Modeling, Identification and Control, Vol. 36, No. 2, 2015, pp. 81-101, ISSN 1890-1328

\title{
Path Generation for High-Performance Motion of ROVs Based on a Reference Model
}

\author{
Daniel de A. Fernandes ${ }^{1}$ Asgeir J. Sørensen ${ }^{1}$ Décio C. Donha ${ }^{2}$
}

\begin{abstract}
${ }^{1}$ Centre for Autonomous Marine Operations and Systems (AMOS), Department of Marine Technology (IMT), Norwegian University of Science and Technology (NTNU), Otto Nielsens veg 10, NO-7491, Trondheim, Norway. E-mails: dalmeidafernandes@gmail.com, \{daniel.fernandes, asgeir.sorensen\}@ntnu.no

${ }^{2}$ Department of Mechanical Engineering (PME), Polytechnic School of the University of São Paulo (Poli/USP), Av. Prof. Mello Moraes, 2231, 05508-900, São Paulo, Brazil. E-mail: decdonha@usp.br
\end{abstract}

\begin{abstract}
This paper deals with the generation of sufficiently smooth position, velocity, and acceleration references for guiding the motion of an ROV along purposefully defined curvature-continuous paths in automated missions. The references are meant to be employed in high-performance trajectory tracking and dynamic positioning applications. The path planning problem is not in the scope of this work. A reference model that synthesises references concerning a single Degree-of-Freedom (DoF) motion is initially described. Then, the use of the synthesised references as the parametrisation for other references concerning multiple DoF motion along curvature-continuous paths is exploited. Results from computer simulations and full-scale sea trials, both based on the NTNU's ROV Minerva, are presented and discussed.
\end{abstract}

Keywords: Guidance system, Path generation, Reference model, ROV, Trajectory tracking.

\section{List of acronyms and abbreviations}

$\begin{array}{ll}(\mathrm{A}) \mathrm{CW} & =\text { Anti)Clockwise } \\ \mathrm{AUV}(\mathrm{s}) & =\text { Autonomous Underwater Vehicle(s) } \\ \mathrm{BF} & =\text { Body-Fixed } \\ \mathrm{CNC} & =\text { Computer Numeric Controlled } \\ \mathrm{DoF}(\mathrm{s}) & =\text { Degree(s)-of-Freedom } \\ \mathrm{DP} & =\text { Dynamic Positioning } \\ \mathrm{DVL} & =\text { Doppler Velocity Log } \\ \mathrm{FBRM}(\mathrm{s}) & =\text { Filter-Based Reference Model(s) } \\ \mathrm{IMU} & =\text { Inertial Measurement Unit } \\ \mathrm{LOS} & =\text { Line-of-Sight } \\ \mathrm{MCS}(\mathrm{s}) & =\text { Motion Control System(s) } \\ \mathrm{MEMS} & =\text { Micro-Electro-Mechanical System } \\ \mathrm{MRAC} & =\text { Model Reference Adaptive Control } \\ \mathrm{NED} & =\text { North-East-Down } \\ \mathrm{RM} & =\text { Reference Model } \\ \text { ROV }(\mathrm{s}) & =\text { Remotely Operated Vehicle(s) } \\ \mathrm{UUV}(\mathrm{s}) & =\text { Unmanned Underwater Vehicle(s) }\end{array}$

\section{Introduction}

This paper deals with the generation of sufficiently smooth position, velocity, and acceleration references for guiding the motion of Remotely Operated Vehicles (ROVs) along purposefully defined curvature-continuous $\left(G^{2}\right)$ paths in automated missions. The references are meant to be employed in high-performance Motion Control Systems (MCSs) with trajectory tracking and Dynamic Positioning (DP) capabilities, e.g. Fernandes et al. (2015), Omerdic et al. (2012), Sørensen et al. (2012), Caccia (2006), and Hsu et al. (2000). Although this work focuses on (typically) fully-actuated Unmanned Underwater Vehicles (UUVs) such as ROVs, some of the ideas presented herein can be adapted to be used as a means of also guiding the motion of (typically) underactuated marine crafts, e.g. ships and UUVs such as Autonomous Underwater Vehicles (AUVs), in path following motion control applications. 
ROVs are indispensable workhorses used worldwide for industrial, research, and military activities, e.g. inspection, intervention, mapping, and survey. They are teleoperated from support surface vessels through umbilical cables which transmit power, commands, and data. Detailed information can be found in e.g. Christ and Wernli (2014, 2007). Granted that accurate motion control is desirable regardless of the type of automated mission that is performed, an MCS with trajectory tracking and DP capabilities has to incorporate a guidance subsystem capable of generating suitable and sufficiently smooth references, given that only the references possessing such attributes can be exactly tracked (Sørensen, 2013; Fossen, 2011; Slotine and Li, 2005).

Guidance is concerned with the transient motion behaviour associated with the achievement of the motion control objectives (Fossen, 2011; Breivik and Fossen, 2009), so that the mission specifications and the vehicle dynamics are all simultaneously observed. Furthermore, collisions with stationary obstacles are avoided whenever a collision-free path is closely tracked or followed. The path planning problem is not in the scope of this work. The reader is referred to Tsourdos et al. (2011), Kavraki and LaValle (2008), Minguez et al. (2008), and LaValle (2006) when it comes to the path planning problem, where the latter provides a thorough coverage of the subject. The former reference is concerned with cooperative path planning of unmanned aerial vehicles, whereas both middle references address the robotics task of planning collision-free motion.

Naeem et al. (2003) reviewed several guidance laws relevant to UUVs, with an emphasis on AUVs, and asserted that 'the main problem in bringing autonomy to any vehicle lies in the design of a suitable guidance law'. Among other conclusions, Naeem et al. (2003) stated that, 'in practice, Line-of-Sight (LoS) guidance is the key element of all guidance systems', given that the closed-loop path following scheme suits best the needs when it comes to guiding underactuated vehicles. That work made evident the fact that the research on guidance has historically been focusing mainly on underactuated vehicles such as missiles and aircrafts, whose complex guidance problems have been dealt with since the World War II (Breivik and Fossen, 2009). Breivik and Fossen (2009) carried out another survey of the subject keeping the emphasis on AUVs, but taking planar and spatial motion into account. An example of early MCS for UUVs based on the LoS guidance is the work by Healey and Lienard (1993). Improvements aiming at compensating for heading disturbances caused by the sea current can be found in e.g. Aguiar and Pascoal (1997). Fossen and Pettersen (2014) proved that the equilibrium point of the proportional LoS guidance law by Healey and Lienard (1993) is Uni- formly Semi-Globally Exponentially Stable (USGES). The work by Caharija et al. (2014) aims at merging intuitive and theoretical perspectives of the integral LoS guidance for current compensation problems of underactuated ships. The technical challenges underactuated vehicles impose on the reference generation, due to their non-holonomic kinematic constraints (Aicardi et al., 2000; Egeland et al., 1996), justify the attention they have received. The LoS and LoS-based guidance laws are still often employed to guide ROVs, e.g. Omerdic et al. (2012), Sørensen et al. (2012), and Caccia (2006), due to their simplicity and ease of implementation (Breivik and Fossen, 2009; Naeem et al., 2003). An example of LoS-based conjoint guidance and control scheme that generates reference heading to steer an ROV towards the destination point employing a Lyapunov-based algorithm to ensure smoothness and convergence is given by Caccia et al. (1998). The approach was refined in Caccia and Veruggio (2000). A similar MCS by Guo et al. (2003), conceived to control the motion of AUVs, employs a sliding mode fuzzy algorithm in place of the Lyapunov-based algorithm. Dukan (2014) proposed a spatial LoS guidance strategy dedicated to guide fully actuated ROVs. The interested reader is referred to Caharija (2014) and Lekkas (2014) when it comes to more recent extensions regarding the LoS and LoS-based guidance laws for underactuated marine vehicles.

ROV-based missions neither typically need high autonomy levels, nor present technical challenges with respect to non-holonomic constraints. On the other hand, repetitive missions, and missions which require accurate motion control, claim some degree of automation. Furthermore, ROV pilots may feel exhausted and less attentive during long-lasting missions, and these factors may lead to riskier and more expensive missions. The reader is referred to Vasilijević et al. (2013), Ho et al. (2011), and Hsu et al. (2000) for discussions about the challenges entailed in ROV piloting. Experienced pilots have affirmed that every contribution towards automating ROV-based missions is effective not only in increasing the overall motion control accuracy level, but also in improving the global mission performance (Hsu et al., 2000). Fully-actuated ROVs permit high-performance motion control to be exercised through trajectory tracking and DP. A collection of guidance techniques, ranging from open-loop Filter-Based Reference Models (FBRMs) to closedloop optimisation-based reference generators, suitable for guiding the motion of fully-actuated UUVs can be found in Fossen (2011) and in the references therein. The simplest, yet effective, technique consists of an FBRM built upon a 2nd- or 3rd-order Low-Pass Filter (LPF) transfer function. It can be easily implemented 
and modified, and runs fast, even in a digital computer with modest hardware. Its drawback lies in its linearity, because, for any fixed tuning, the generated velocities and accelerations are linearly proportional to the distances to be covered (Fernandes et al., 2012; Fossen, 2011). Fossen (2011) presents alternatives to partially remedy the problem. Linear FBRMs stem from the Model Reference Adaptive Control (MRAC) technique (Landau, 1974), and are commonly employed in trajectory tracking control systems to improve the closed-loop transient response (Aström and Hägglund, 2011; Slotine and Li, 2005).

This paper makes two contributions to the literature on guidance:

- A Reference Model (RM) that generates references for steady single Degree-of-Freedom (DoF) motion and is easy, i.e. meaningful, to tune;

- A path generation (reference generation) scheme, built upon the RM, that generates references for steady multiple DoF motion.

The motivation for developing the RM was to develop another alternative to the useful, yet simple, FBRM found in e.g. Sørensen (2013) and Fossen (2011). The inspiration that underlies the development came from works on motion optimisation for AUVs, e.g. Chyba et al. (2008) and Kumar et al. (2005), although this work does not necessarily seek to provide optimal references, and from works on reference generation for highly accurate Computer Numeric Controlled (CNC) machines, e.g. Huo and Poo (2012) and Matsubara et al. (2011). It is important to emphasise that the references are generated in open-loop through an FBRM in Matsubara et al. (2011), whereas they are directly synthesised by computers in Huo and Poo (2012). The proposed RM synthesises references for guiding a single DoF motion, either linear or angular, in a suboptimal manner with regard to time. The maximum - or minimum, depending on the motion direction - velocity is kept for the longest time span possible, as exemplified in Figure 1. Such velocity regime is intended to: i) induce steadier hydrodynamic effects; and ii) demand steadier thrust forces and moments from the ROV's propulsion system. Both factors together favour the attainment of small reference tracking errors, and also provide more favourable conditions for adaptive controllers and/or observers, if any, to faster and more robustly estimate and cope with the unknown current-generated perturbations. The RM generates references via direct computer synthesis, similarly to Huo and Poo (2012). Thus, repeatability, finite convergence time, and facilitated interaction with the references while they are being synthesised, e.g. starting, pausing, resuming, and aborting, can be achieved. The shapes of the synthesised references

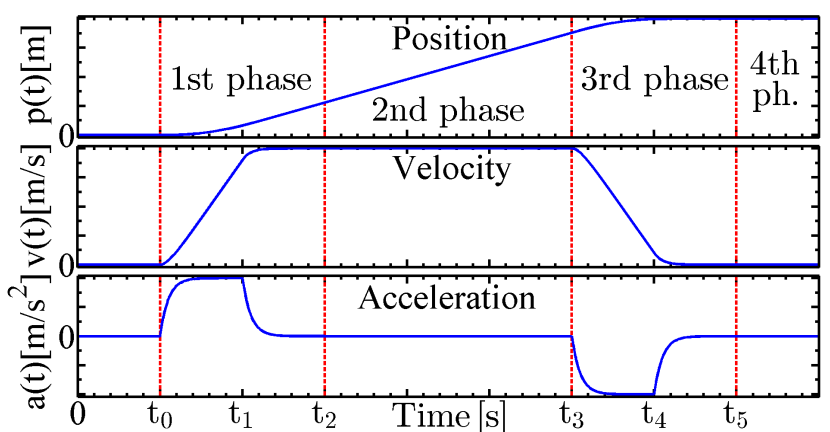

Figure 1: Shapes of the references synthesised by the RM concerning a single DoF motion.

keep much resemblance with those considered better in Kumar et al. (2005). The present work is an extension of that reported in Fernandes et al. (2012). The computer code of the RM has been improved. It is currently more efficient and needs less memory space to be stored than before.

The path generation scheme utilises the references synthesised by the RM as the parametrisation of a group of references which are generated for guiding the four DoF - surge, sway, heave, and yaw — motion of an ROV along pre-defined curvature-continuous paths.

The rest of the paper is organised as follows. Section 2 deals with the RM. Section 3 deals with the path generation scheme. Section 4 presents selected results from simulations and full-scale sea trials, both based on the NTNU's ROV Minerva, which is introduced in Appendix B. Section 5 presents concluding remarks. Appendix A presents the optimal curve shapes (single DoF) along a rectilinear path under the condition of constrained acceleration and velocity.

\section{Reference model}

\subsection{Description of the basic version}

The RM synthesises position, velocity, and acceleration references of classes $\mathcal{C}^{2}, \mathcal{C}^{1}$, and $\mathcal{C}^{0}$, respectively, for guiding a single DoF motion in a suboptimal manner with regard to time. The differentiability class $\mathcal{C}^{n}$, where $n \in \mathbb{N}$, denotes a function whose $n$-th derivative with respect to time exists and is continuous on the domain of definition of the function. The condition of suboptimality has harmless implications in practice. It is mostly a consequence of the fact that the RM has to reflect the constraints associated with the dynamics of the guided ROV, e.g. limited acceleration and velocity. This point becomes clearer when the shapes of the references synthesised by the RM, which are exemplified in Figure 1, are compared with the optimal curve shapes shown and discussed in Appendix A. The 
references are parameterised by the parameter time $t \in$ $\mathbb{R}_{\geqslant 0}[\mathrm{~s}]$. They are synthesised through a combination of functions distributed into four consecutive phases, as seen in Figure 1, where each function is active only during a certain amount of time, such that

$$
\begin{aligned}
a(t) & :=a_{1}(t)+a_{2}(t)+a_{3}(t)+a_{4}(t), \\
v(t) & :=v_{1}(t)+v_{2}(t)+v_{3}(t)+v_{4}(t),
\end{aligned}
$$

and

$$
p(t):=p_{1}(t)+p_{2}(t)+p_{3}(t)+p_{4}(t),
$$

where $a(t)\left[\mathrm{m} / \mathrm{s}^{2}\right], v(t)[\mathrm{m} / \mathrm{s}]$, and $p(t)[\mathrm{m}]$ denote the acceleration, velocity, and position references, respectively. The subscripts ' 1 ' through ' 4 ' identify the phases. Every phase is separately described in Subsubsections 2.1.1-2.1.4. The required pre-computations are introduced in Subsubsection 2.1.5. Each tuning parameter is explained where mentioned for the first time. Table 1 collects and summarises all tuning parameters in advance. They are divided into two categories. One category includes the tuning parameters which are frequently readjusted to satisfy the application needs the first six table entries. The third and fourth tuning parameters are meaningless with regard to the basic version of the RM. The second category includes the last four table entries. These tuning parameters hardly ever require readjustments, once they are satisfactorily tuned for a particular use.

Remark: A linear motion has been chosen to be used in the explanation given throughout Section 2, without loss of generality. Should an angular motion be guided, it is enough to consider $L$ in 'rad' or 'o' (degree), and $V_{d}$ in 'rad/s' or '\%/s', in Table 1.

\subsubsection{First phase: the acceleration phase}

This phase is characterised by the modulus of the velocity reference increasing from zero to virtually the maximum value, see Figure 1. The phase is split into two subphases in which the modulus of the acceleration reference firstly increases from zero to virtually the maximum absolute acceleration, and thence decreases to virtually zero again. During this phase, the references are defined as

$$
\begin{aligned}
a_{1}(t):= & a_{m} \mathrm{f}_{11}(t) \mathrm{h}_{11}(t)+a_{m} \mathrm{f}_{12}(t) \mathrm{h}_{12}(t), \\
v_{1}(t):= & a_{m}\left(\left(t-t_{0}\right)-\tau_{11} \mathrm{f}_{11}(t)\right) \mathrm{h}_{11}(t) \\
& +v_{m}\left(1-\left(1-\theta_{a}\right) \mathrm{f}_{12}(t)\right) \mathrm{h}_{12}(t),
\end{aligned}
$$

and

$$
\begin{aligned}
p_{1}(t):= & a_{m}\left(\left(t-t_{0}\right)^{2} / 2-\tau_{11}\left(t-t_{0}\right)+\tau_{11}^{2} \mathrm{f}_{11}(t)\right) \mathrm{h}_{11}(t) \\
& +v_{m}\left(\left(t-t_{1}\right)-\left(1-\theta_{a}\right) \tau_{12}\left(1-\mathrm{f}_{12}(t)\right)\right) \mathrm{h}_{12}(t) \\
& +P_{1} \mathrm{~h}_{12}(t)
\end{aligned}
$$

Table 1: Tuning parameters of the RM

\begin{tabular}{lll}
\hline$L$ & Path length (straight line) & {$[\mathrm{m}]$} \\
$V_{d}$ & Desired cruise velocity & {$[\mathrm{m} / \mathrm{s}]$} \\
$V_{i}$ & Des. initial vel. (advanced version only) & {$[\mathrm{m} / \mathrm{s}]$} \\
$V_{f}$ & Des. final vel. (advanced version only) & {$[\mathrm{m} / \mathrm{s}]$} \\
$T_{a}$ & Desired minimum time to reach $V_{d}$ & {$[\mathrm{~s}]$} \\
$T_{d}$ & Desired minimum time to stop from $V_{d}$ & {$[\mathrm{~s}]$} \\
\hline$\epsilon_{L}$ & Min. fraction of $L$ to be traversed at $v_{m}$ & {$[-]$} \\
$\theta_{a}$ & Function switching threshold & {$[-]$} \\
$\theta_{d}$ & Function switching threshold & {$[-]$} \\
$\theta_{0}$ & Function switching threshold & {$[-]$} \\
\hline
\end{tabular}

where $a_{m} \in \mathbb{R}\left[\mathrm{m} / \mathrm{s}^{2}\right]$ is the maximum - or minimum, depending on the motion direction - acceleration, $v_{m} \in \mathbb{R}[\mathrm{m} / \mathrm{s}]$ is the maximum - or minimum, depending on the motion direction - velocity, $\tau_{11}, \tau_{12} \in$ $\mathbb{R}_{>0}[\mathrm{~s}]$ are time constants respectively associated with the 1 st and 2 nd subphases, $t_{0} \in \mathbb{R}_{\geqslant 0}[\mathrm{~s}]$ is the instant at which this phase begins, and $t_{1} \in \mathbb{R}_{>0} \mid t_{1}>t_{0}[\mathrm{~s}]$ is the instant at which the 2 nd subphase begins, when the velocity reference reaches the threshold $v_{1}\left(t_{1}\right)=$ $v_{m} \theta_{a}[\mathrm{~m} / \mathrm{s}]$, where $\theta_{a} \in \mathbb{R} \mid \theta_{a} \in[0.6,1)$ is the tuning parameter (see Table 1 ) that defines the fraction of $v_{m}$ at which the velocity reference starts getting bent as it proceeds towards $v_{m}$. Lastly, $P_{1} \in \mathbb{R} \mid P_{1}:=p_{1}\left(t_{1}^{-}\right)$ $[\mathrm{m}]$, where $t_{1}^{-}$denotes $t \nearrow t_{1}$, i.e. parameter $t$ tending to $t_{1}$ from the left. The expressions of $a_{m}, v_{m}, \tau_{11}$, $\tau_{12}, t_{1}$, and $P_{1}$ are given in Subsubsection 2.1.5. The auxiliary functions $\mathrm{f}_{11}(t)$ and $\mathrm{f}_{12}(t)$ are defined as

$$
\mathrm{f}_{11}(t):=1-\exp \left(-\left(t-t_{0}\right) / \tau_{11}\right)
$$

and

$$
\mathrm{f}_{12}(t):=\exp \left(-\left(t-t_{1}\right) / \tau_{12}\right) .
$$

It is worthwhile to realise that $(7)-(8)$ can virtually make $\lim _{t \rightarrow t_{1}} a_{1}(t)=a_{m}\left[\mathrm{~m} / \mathrm{s}^{2}\right]$, i.e. virtually render (4) continuous. The functions $\mathrm{h}_{11}(t)$ and $\mathrm{h}_{12}(t)$, which are the characteristic functions of the half-closed finite intervals $\left[t_{0}, t_{1}\right)$ and $\left[t_{1}, t_{2}\right)$, respectively, are defined as

$$
\mathrm{h}_{11}(t):=\mathrm{H}\left(t-t_{0}\right)-\mathrm{H}\left(t-t_{1}\right),
$$

and

$$
\mathrm{h}_{12}(t):=\mathrm{H}\left(t-t_{1}\right)-\mathrm{H}\left(t-t_{2}\right),
$$

where $\mathrm{H}(\cdot)$ is the unit step function, and $t_{2} \in \mathbb{R}_{>0} \mid t_{2}>$ $t_{1}[\mathrm{~s}]$ is the instant at which this phase ends, when the acceleration reference reaches the threshold $a_{1}\left(t_{2}^{-}\right):=$ $\theta_{0} a_{m}$, beyond which the acceleration reference virtually vanishes, where $t_{2}^{-}$denotes $t \nearrow t_{2}$, i.e. parameter $t$ tending to $t_{2}$ from the left, and $\theta_{0} \in \mathbb{R}_{>0} \mid \theta_{0} \ll 1$ is another tuning parameter (see Table 1 ), which is discussed within the next subsubsection. The expression

(6) of $t_{2}$ is furnished in Subsubsection 2.1.5. 


\subsubsection{Second phase: the constant velocity phase}

This phase is characterised by the (constant) cruise velocity $v_{m}$, see Figure 1 . During this phase, the references are defined as

$$
\begin{gathered}
a_{2}(t):=0 \mathrm{~h}_{2}(t), \\
v_{2}(t):=v_{m} \mathrm{~h}_{2}(t),
\end{gathered}
$$

and

$$
p_{2}(t):=\left(P_{2}+v_{m}\left(t-t_{2}\right)\right) \mathrm{h}_{2}(t),
$$

where $P_{2} \in \mathbb{R} \mid P_{2}:=p_{1}\left(t_{2}^{-}\right)[\mathrm{m}]$. The expression of $P_{2}$ is furnished in Subsubsection 2.1.5. The function $\mathrm{h}_{2}(t)$, which is the characteristic function of the half-closed finite interval $\left[t_{2}, t_{3}\right)$, is defined as

$$
\mathrm{h}_{2}(t):=\mathrm{H}\left(t-t_{2}\right)-\mathrm{H}\left(t-t_{3}\right) .
$$

Remark: Every reference undergoes a step discontinuity of negligibly small magnitude at the transition from the 1 st phase to the 2 nd phase due to the presence of the exponential function in (8). The lesser $\theta_{0}$, the lesser the magnitudes of the step discontinuities. On the other hand, the lesser $\theta_{0}$, the longer the 1 st phase lasts, hence causing the cruise velocity $v_{m}$ to be reduced. For instance, if $\theta_{0}=\exp (-7)$ and $a_{m}=1 \mathrm{~m} / \mathrm{s}^{2}$, then $a\left(t_{2}\right)$ undergoes a step of magnitude $\left|\theta_{0} a_{m}\right|<9.12 \times$ $10^{-4} \mathrm{~m} / \mathrm{s}^{2}(<0.1 \%)$. Likewise, if $v_{m}=1 \mathrm{~m} / \mathrm{s}$, then $v\left(t_{2}\right)$ undergoes a step of magnitude $\left(1-\theta_{a}\right) \theta_{0} v_{m}<$ $9.12 \times 10^{-4} \mathrm{~m} / \mathrm{s}(<0.1 \%)$. Likewise, if $\tau_{12}=1 \mathrm{~s}$, then $p\left(t_{2}\right)$ undergoes a step of magnitude $\left(1-\theta_{a}\right) \theta_{0} \tau_{12}<$ $9.12 \times 10^{-4} \mathrm{~m}$. If $\theta_{0}=\exp (-11.11) \approx 1.5 \times 10^{-5}$ instead, whereas all other values are kept the same as before, the step discontinuities would virtually disappear in the face of quantisation (Åström and Wittenmark, 1997), if the RM was implemented in a 16-bit digital system, given that $2^{-16} \approx 1.53 \times 10^{-5}$ yields the resolution of $\approx 0.0015 \%$. Therefore, in this work, it is defined that $\exp (-13) \leqslant \theta_{0} \leqslant \exp (-7)$.

\subsubsection{Third phase: the deceleration phase}

This phase is characterised by the modulus of the velocity reference decreasing from the maximum value, i.e. $\left|v_{m}\right|$, to virtually zero, see Figure 1 . The phase is split into two subphases in which the modulus of the acceleration reference firstly increases from zero to virtually the maximum absolute deceleration, and thence decreases to virtually zero again. During this phase, the references are defined as

$$
\begin{aligned}
a_{3}(t): & =d_{m} \mathrm{f}_{31}(t) \mathrm{h}_{31}(t)+d_{m} \mathrm{f}_{32}(t) \mathrm{h}_{32}(t), \\
v_{3}(t):= & \left(v_{m}+d_{m}\left(\left(t-t_{3}\right)-\tau_{31} \mathrm{f}_{31}(t)\right)\right) \mathrm{h}_{31}(t) \\
& +v_{m} \theta_{d} \mathrm{f}_{32}(t) \mathrm{h}_{32}(t),
\end{aligned}
$$

and

$$
\begin{aligned}
p_{3}(t):= & \left(P_{3}+v_{m}\left(t-t_{3}\right)\right) \mathrm{h}_{31}(t) \\
& +d_{m}\left(\left(t-t_{3}\right)^{2} / 2-\tau_{31}\left(t-t_{3}\right)+\tau_{31}^{2} \mathrm{f}_{31}(t)\right) \mathrm{h}_{31}(t) \\
& +\left(P_{4}+v_{m} \theta_{d} \tau_{32}\left(1-\mathrm{f}_{32}(t)\right)\right) \mathrm{h}_{32}(t),
\end{aligned}
$$

where $d_{m} \in \mathbb{R}\left[\mathrm{m} / \mathrm{s}^{2}\right]$ is the maximum - or minimum, depending on the motion direction - deceleration, $\tau_{31}, \tau_{32} \in \mathbb{R}_{>0}[\mathrm{~s}]$ are time constants respectively associated with the 1 st and 2 nd subphases, $t_{3} \in \mathbb{R}_{>0} \mid t_{3}>t_{2}$ $[\mathrm{s}]$ is the instant at which this phase begins, $t_{4} \in \mathbb{R}_{>0} \mid$ $t_{4}>t_{3}[\mathrm{~s}]$ is the instant at which the 2nd subphase begins, when the velocity reference reaches the threshold $v_{3}\left(t_{4}\right)=v_{m} \theta_{d}[\mathrm{~m} / \mathrm{s}]$, where $\theta_{d} \in \mathbb{R} \mid \theta_{d} \in(0,0.4]$ is the tuning parameter (see Table 1) that defines the fraction of $v_{m}$ at which the velocity reference starts getting bent as it proceeds towards zero. Lastly, $P_{3} \in$ $\mathbb{R} \mid P_{3}:=p_{2}\left(t_{3}^{-}\right)[\mathrm{m}]$, where $t_{3}^{-}$denotes $t \nearrow t_{3}$, i.e. parameter $t$ tending to $t_{3}$ from the left, and $P_{4} \in \mathbb{R} \mid P_{4}:=$ $p_{3}\left(t_{4}^{-}\right)[\mathrm{m}]$, where $t_{4}^{-}$denotes $t \nearrow t_{4}$, i.e. parameter $t$ tending to $t_{4}$ from the left. The expressions of $d_{m}, \tau_{31}$, $\tau_{32}, t_{3}, t_{4}, P_{3}$, and $P_{4}$ are given in Subsubsection 2.1.5. The auxiliary functions $\mathrm{f}_{31}(t)$ and $\mathrm{f}_{32}(t)$ are defined as

$$
\mathrm{f}_{31}(t):=1-\exp \left(-\left(t-t_{3}\right) / \tau_{31}\right)
$$

and

$$
\mathrm{f}_{32}(t):=\exp \left(-\left(t-t_{4}\right) / \tau_{32}\right) .
$$

It is worthwhile to realise that (18)-(19) can virtually make $\lim _{t \rightarrow t_{4}} a_{3}(t)=d_{m}\left[\mathrm{~m} / \mathrm{s}^{2}\right]$, i.e. virtually render (15) continuous. The functions $\mathrm{h}_{31}(t)$ and $\mathrm{h}_{32}(t)$, which are the characteristic functions of the half-closed finite intervals $\left[t_{3}, t_{4}\right)$ and $\left[t_{4}, t_{5}\right)$, respectively, are defined as

$$
\mathrm{h}_{31}(t):=\mathrm{H}\left(t-t_{3}\right)-\mathrm{H}\left(t-t_{4}\right),
$$

and

$$
\mathrm{h}_{32}(t):=\mathrm{H}\left(t-t_{4}\right)-\mathrm{H}\left(t-t_{5}\right) .
$$

\subsubsection{Fourth phase: the constant position phase}

This phase is characterised by the constant position reference, see Figure 1. During this phase, the references are defined as

$$
\begin{aligned}
& a_{4}(t):=0 \mathrm{~h}_{4}(t), \\
& v_{4}(t):=0 \mathrm{~h}_{4}(t),
\end{aligned}
$$

and

$$
p_{4}(t):=\operatorname{sgn}\left(v_{m}\right) L \mathrm{~h}_{4}(t),
$$

where $L \in \mathbb{R}_{>0}[\mathrm{~m}]$ is the tuning parameter (see Table 1) that defines the (straight line) path length. The 
function $\mathrm{h}_{4}(t)$, which is the characteristic function of the closed semi-infinite interval $\left[t_{5}, \infty\right)$, is defined as

$$
\mathrm{h}_{4}(t):=\mathrm{H}\left(t-t_{5}\right)
$$

Remark: Every reference undergoes another step discontinuity of negligibly small magnitude at the transition from the 3rd phase to the 4th phase due to the presence of the exponential function in (19).

\subsubsection{Pre-computation}

Let the time ratio $r_{T} \in \mathbb{R}_{>0}$ be defined as

$$
r_{T}:=T_{a} / T_{d}
$$

where $T_{a} \in \mathbb{R}_{>0}[\mathrm{~s}]$ is the tuning parameter (see Table 1 ) that defines the desired minimum time to reach $V_{d} \in$ $\mathbb{R} \backslash\{0\}[\mathrm{m} / \mathrm{s}]$, which is the tuning parameter (see Table 1) that defines the desired cruise velocity, and $T_{d} \in$ $\mathbb{R}_{>0}[\mathrm{~s}]$ is the tuning parameter (see Table 1 ) that defines the desired minimum time to stop moving from $V_{d}$. These parameters translate the desired maximum — or minimum, depending on the motion direction acceleration and deceleration through the direct relations ' $V_{d} / T_{a}$ ' and ' $-V_{d} / T_{d}$ ', respectively.

Let the auxiliary constants $\kappa_{a}, \kappa_{d} \in \mathbb{R}_{>0}$ be defined as

$$
\begin{aligned}
\kappa_{a}:= & \frac{\theta_{a}^{2}\left(\left(\xi_{a}-\exp \left(-\xi_{a}\right)\right)^{2}-2\left(\xi_{a}-1\right)\right)}{2\left(\xi_{a}-1\right)^{2}} \\
& -\left(1-\theta_{a}\right)^{2}\left(1-\theta_{0}\right)-\left(1-\theta_{a}\right) \ln \left(\theta_{0}\right),
\end{aligned}
$$

and

$$
\begin{aligned}
\kappa_{d}:= & -\frac{\left(1-\theta_{d}\right)^{2}\left(\xi_{d}-\exp \left(-\xi_{d}\right)\right)^{2}}{2\left(\xi_{d}-1\right)^{2}}+\theta_{d}^{2}\left(1-\theta_{0}\right) \\
& +\frac{\left(1-\theta_{d}\right)^{2}+\left(1-\theta_{d}\right)\left(\xi_{d}-\exp \left(-\xi_{d}\right)\right)}{\xi_{d}-1},
\end{aligned}
$$

where $\xi_{a}, \xi_{d} \in \mathbb{R}_{>0}$ are constants. The greater $\xi_{a}$ and $\xi_{d}$, the steeper the slopes of the references during the 1 st subphases of the 1st and 3rd phases, respectively, since these constants directly influence the time constants defined in (35) and (37) ahead. Even though $\xi_{a}$ and $\xi_{d}$ are not primarily designed to be tuning parameters, it can be useful to be able to change their values. Hence, it is defined that $10 \leqslant \xi_{a}, \xi_{d} \leqslant 15$ in this work.

Then, the candidate absolute value $v_{c} \in \mathbb{R}_{>0}[\mathrm{~m} / \mathrm{s}]$ for the cruise velocity is defined as

$$
v_{c}:=\sqrt{L\left(\frac{1-\min \left\{\epsilon_{L}, 0.1\right\}}{\kappa_{a} r_{T}+\kappa_{d}}\right) \frac{\left|V_{d}\right|}{T_{d}}},
$$

where $\epsilon_{L} \in \mathbb{R}_{>0} \mid \epsilon_{L}<0.1$ is the tuning parameter (see Table 1) that defines the minimum fraction of the path length $L$ that is to be traversed at the cruise velocity $v_{m}$, which is defined as

$$
v_{m}:=\operatorname{sgn}\left(V_{d}\right) \min \left\{v_{c},\left|V_{d}\right|\right\} .
$$

The adjusted minimum time to stop moving from $v_{m}$, i.e. $t_{d} \in \mathbb{R}_{>0}[\mathrm{~s}]$, and the adjusted minimum time to reach $v_{m}$, i.e. $t_{a} \in \mathbb{R}_{>0}[\mathrm{~s}]$, are defined as

$$
t_{d}:=\left(v_{m} / V_{d}\right) T_{d}
$$

and

$$
t_{a}:=r_{T} t_{d}
$$

The maximum (minimum) acceleration and deceleration are defined as

$$
a_{m}:=v_{m} / t_{a},
$$

and

$$
d_{m}:=-\left(v_{m} / t_{d}\right)
$$

The time constants $\tau_{11}$ through $\tau_{32}$ are defined as

$$
\begin{gathered}
\tau_{11}:=\theta_{a} t_{a} /\left(\xi_{a}-1\right), \\
\tau_{12}:=\left(1-\theta_{a}\right) t_{a}, \\
\tau_{31}:=\left(1-\theta_{d}\right) t_{d} /\left(\xi_{d}-1\right),
\end{gathered}
$$

and

$$
\tau_{32}:=\theta_{d} t_{d}
$$

The auxiliary time instants $T_{i} \in \mathbb{R}_{>0}[\mathrm{~s}], i \in\{1,2,3$, $4,5\}$, where $T_{i}:=t_{i}^{-}$, are defined as

$$
\begin{gathered}
T_{1}:=\xi_{a} \tau_{11}, \\
T_{2}:=-\tau_{12} \ln \left(\theta_{0}\right), \\
T_{3}:=\left|L / v_{m}\right|-\left(\kappa_{a} t_{a}+\kappa_{d} t_{d}\right), \\
T_{4}:=\xi_{d} \tau_{31},
\end{gathered}
$$

and

$$
T_{5}:=-\tau_{32} \ln \left(\theta_{0}\right),
$$

such that the function switching time instants $t_{i}, i \in$ $\{1,2,3,4,5\}$, are consecutively defined, with respect to the initial instant $t_{0}$, as

$$
t_{i}:=T_{i}+t_{(i-1)} .
$$

Finally, the positions $P_{i}, i \in\{1,2,3,4\}$, are respectively defined as

$$
\begin{gathered}
P_{1}:=a_{m}\left(T_{1}^{2} / 2-\tau_{11} T_{1}+\tau_{11}^{2}\left(1-\exp \left(-\xi_{a}\right)\right)\right), \\
P_{2}:=P_{1}+v_{m}\left(T_{2}-\left(1-\theta_{a}\right) \tau_{12}\left(1-\theta_{0}\right)\right), \\
P_{3}:=P_{2}+v_{m} T_{3},
\end{gathered}
$$

and

$P_{4}:=P_{3}+v_{m} T_{4}$

$$
+d_{m}\left(T_{4}^{2} / 2-\tau_{31} T_{4}+\tau_{31}^{2}\left(1-\exp \left(-\xi_{d}\right)\right)\right),
$$

where the relation $T_{1} / \tau_{11}=\xi_{a}$, which is used in (45), stems from (39), the relation $T_{2} / \tau_{12}=-\ln \left(\theta_{0}\right)$, which is used in (46), stems from (40), and the relation $T_{4} / \tau_{31}$ $=\xi_{d}$, which is used in (48), stems from (42). 


\subsubsection{Results from full-scale sea trials}

See Subsection 4.1 for the results.

\subsection{Description of an advanced version}

The advanced version builds upon the basic version described in Subsection 2.1 and, thus, it bears strong resemblance to that version. The synthesised references are likewise structured as in (1)-(3). Each of the four phases is separately described in Subsubsections 2.2.12.2.4. The required pre-computations are introduced in Subsubsection 2.2.5. Table 1 collects and summarises all tuning parameters. The main differences in comparison with the basic version are the following:

- the initial velocity and the final velocity can be both different from zero;

- the first and third phases are skipped depending on the initial and final velocities; the second phase is always executed; and the fourth phase is executed whenever the final velocity is null;

- the cruise velocity is numerically determined.

\subsubsection{First phase: the acceleration phase}

This phase is characterised by the modulus of the velocity reference increasing from the modulus of the initial value, i.e. $\left|v_{i}\right|=\left|v\left(t_{0}\right)\right|$, where $v_{i} \in \mathbb{R}[\mathrm{m} / \mathrm{s}]$ is derived from $V_{i} \in \mathbb{R} \mid V_{i}=0 \vee\left(\left|V_{i}\right| \leqslant\left|V_{d}\right| \wedge \operatorname{sgn}\left(V_{i}\right)=\right.$ $\left.\operatorname{sgn}\left(V_{d}\right)\right)[\mathrm{m} / \mathrm{s}]$, which is the tuning parameter (see Table 1) that defines the desired initial velocity, to virtually the maximum value, i.e. $\left|v_{m}\right|=\left|v\left(t_{2}\right)\right|$, where $v_{m} \in \mathbb{R}[\mathrm{m} / \mathrm{s}]$ is the cruise velocity. This phase is skipped whenever $v_{i}=v_{m}$. Likewise in the basic version, this phase is split into two subphases. During this phase, the references are defined as

$$
\begin{gathered}
a_{1}(t):=a_{m} \mathrm{f}_{11}(t) \mathrm{h}_{11}(t)+a_{m} \mathrm{f}_{12}(t) \mathrm{h}_{12}(t), \\
v_{1}(t):=\left(v_{i}+a_{m}\left(\left(t-t_{0}\right)-\tau_{11} \mathrm{f}_{11}(t)\right)\right) \mathrm{h}_{11}(t) \\
\quad+\left(v_{m}-\left(v_{m}-v_{i}\right)\left(1-\theta_{a}\right) \mathrm{f}_{12}(t)\right) \mathrm{h}_{12}(t),
\end{gathered}
$$

and

$$
\begin{aligned}
p_{1}(t):= & \left(v_{i}-a_{m} \tau_{11}\right)\left(t-t_{0}\right) \mathrm{h}_{11}(t) \\
& +a_{m}\left(\left(t-t_{0}\right)^{2} / 2+\tau_{11}^{2} \mathrm{f}_{11}(t)\right) \mathrm{h}_{11}(t) \\
& +\left(P_{1}+v_{m}\left(t-t_{1}\right)\right) \mathrm{h}_{12}(t) \\
& -\left(v_{m}-v_{i}\right)\left(1-\theta_{a}\right) \tau_{12}\left(1-\mathrm{f}_{12}(t)\right) \mathrm{h}_{12}(t),
\end{aligned}
$$

where $a_{m} \in \mathbb{R}\left[\mathrm{m} / \mathrm{s}^{2}\right]$ is the maximum - or minimum, depending on the motion direction - acceleration, which is computed as indicated in (33), $\tau_{11}, \tau_{12} \in \mathbb{R}_{>0}$ $[\mathrm{s}]$ are time constants respectively associated with the 1st and 2nd subphases, $t_{0} \in \mathbb{R}_{\geqslant 0}[\mathrm{~s}]$ is the instant at which this phase begins, and $t_{1} \in \mathbb{R}_{\geqslant 0} \mid t_{1} \geqslant t_{0}[\mathrm{~s}]$ is the instant at which the 2 nd subphase begins, when the velocity reference reaches the threshold $v_{1}\left(t_{1}\right)=$ $v_{i}+\left(v_{m}-v_{i}\right) \theta_{a}[\mathrm{~m} / \mathrm{s}]$, where $\theta_{a} \in \mathbb{R} \mid \theta_{a} \in[0.6,1)$ is the tuning parameter (see Table 1 ) that defines the fraction of $\left(v_{m}-v_{i}\right)$ at which the velocity reference starts getting bent as it proceeds towards $v_{m}$. Lastly, $P_{1} \in$ $\mathbb{R} \mid P_{1}:=p_{1}\left(t_{1}^{-}\right)[\mathrm{m}]$. The expressions of $v_{i}, v_{m}, \tau_{11}, \tau_{12}$, $t_{1}, P_{1}$, and $t_{a}$, the latter being useful to compute $a_{m}$ as in (33), are given in Subsubsection 2.2.5. The functions $\mathrm{f}_{11}(t), \mathrm{f}_{12}(t), \mathrm{h}_{11}(t)$, and $\mathrm{h}_{12}(t)$ are defined similarly to those in (7)-(10).

\subsubsection{Second phase: the constant velocity phase}

This phase is characterised by the cruise velocity $v_{m}$. During this phase, the references are defined as

$$
a_{2}(t):=0 \mathrm{~h}_{2}(t)
$$

$$
v_{2}(t):=v_{m} \mathrm{~h}_{2}(t)
$$

and

$$
p_{2}(t):=\left(P_{2}+v_{m}\left(t-t_{2}\right)\right) \mathrm{h}_{2}(t)
$$

where $P_{2} \in \mathbb{R} \mid P_{2}:=p_{1}\left(t_{2}^{-}\right)[\mathrm{m}]$, and $t_{2} \in \mathbb{R}_{\geqslant 0} \mid t_{2} \geqslant t_{1}[\mathrm{~s}]$ is the instant at which this phase begins. The expressions of $P_{2}$ and $t_{2}$ are furnished in Subsubsection 2.2.5. The function $h_{2}(t)$ is defined similarly to that in (14).

Notice the similitude between this phase and the second phase of the basic version in Subsubsection 2.1.2.

\subsubsection{Third phase: the deceleration phase}

This phase is characterised by the modulus of the velocity reference decreasing from the maximum value, i.e. $\left|v_{m}\right|=\left|v\left(t_{3}\right)\right|$, to virtually the modulus of the final value, i.e. $\left|v_{f}\right|=\left|v\left(t_{5}\right)\right|$, where $v_{f} \in \mathbb{R}[\mathrm{m} / \mathrm{s}]$ is derived from $V_{f} \in \mathbb{R} \mid V_{f}=0 \vee\left(\left|V_{f}\right| \leqslant\left|V_{d}\right| \wedge \operatorname{sgn}\left(V_{f}\right)=\right.$ $\left.\operatorname{sgn}\left(V_{d}\right)\right)[\mathrm{m} / \mathrm{s}]$, which is the tuning parameter (see Table 1) that defines the desired final velocity. This phase is skipped whenever $v_{f}=v_{m}$. Likewise in the basic version, this phase is split into two subphases. During this phase, the references are defined as

$$
\begin{aligned}
a_{3}(t): & =d_{m} \mathrm{f}_{31}(t) \mathrm{h}_{31}(t)+d_{m} \mathrm{f}_{32}(t) \mathrm{h}_{32}(t), \\
v_{3}(t):= & \left(v_{m}+d_{m}\left(\left(t-t_{3}\right)-\tau_{31} \mathrm{f}_{31}(t)\right)\right) \mathrm{h}_{31}(t) \\
& +\left(v_{f}+\left(v_{m}-v_{f}\right) \theta_{d} \mathrm{f}_{32}(t)\right) \mathrm{h}_{32}(t),
\end{aligned}
$$


and

$$
\begin{aligned}
p_{3}(t):= & \left(P_{3}+v_{m}\left(t-t_{3}\right)\right) \mathrm{h}_{31}(t) \\
& +d_{m}\left(\left(t-t_{3}\right)^{2} / 2-\tau_{31}\left(t-t_{3}\right)+\tau_{31}^{2} \mathrm{f}_{31}(t)\right) \mathrm{h}_{31}(t) \\
& +\left(P_{4}+v_{f}\left(t-t_{4}\right)\right) \mathrm{h}_{32}(t) \\
& +\left(v_{m}-v_{f}\right) \theta_{d} \tau_{32}\left(1-\mathrm{f}_{32}(t)\right) \mathrm{h}_{32}(t)
\end{aligned}
$$

where $d_{m} \in \mathbb{R}\left[\mathrm{m} / \mathrm{s}^{2}\right]$ is the maximum - or minimum, depending on the motion direction - deceleration, which is computed as indicated in (34), $\tau_{31}, \tau_{32} \in \mathbb{R}_{>0}$ $[\mathrm{s}]$ are time constants respectively associated with the 1 st and 2nd subphases, $t_{3} \in \mathbb{R}_{>0} \mid t_{3}>t_{2}[\mathrm{~s}]$ is the instant at which this phase begins, and $t_{4} \in \mathbb{R}_{>0} \mid t_{4} \geqslant t_{3}$ $[\mathrm{s}]$ is the instant at which the 2 nd subphase begins, when the velocity reference reaches the threshold $v_{3}\left(t_{4}\right)$ $=v_{f}+\left(v_{m}-v_{f}\right) \theta_{d}[\mathrm{~m} / \mathrm{s}]$, where $\theta_{d} \in \mathbb{R} \mid \theta_{d} \in(0,0.4]$ is the tuning parameter (see Table 1 ) that defines the fraction of $\left(v_{m}-v_{f}\right)$ at which the velocity reference starts getting bent as it proceeds towards $v_{f}$. Lastly, $P_{3} \in \mathbb{R} \mid P_{3}:=p_{2}\left(t_{3}^{-}\right)[\mathrm{m}]$, and $P_{4} \in \mathbb{R} \mid P_{4}:=p_{3}\left(t_{4}^{-}\right)[\mathrm{m}]$. The expressions of $d_{m}, v_{f}, \tau_{31}, \tau_{32}, t_{3}, t_{4}, P_{3}, P_{4}$, and $t_{d}$, the latter being useful to compute $d_{m}$ as indicated in (34), are given in Subsubsection 2.2.5. The functions $\mathrm{f}_{31}(t), \mathrm{f}_{32}(t), \mathrm{h}_{31}(t)$, and $\mathrm{h}_{32}(t)$ are defined similarly to those in (18)-(21).

\subsubsection{Fourth phase: the constant position phase}

This phase is characterised by the constant position reference. It is executed whenever $v_{f}=0 \mathrm{~m} / \mathrm{s}$. During this phase, the references are defined as

$$
\begin{gathered}
a_{4}(t):=0 \mathrm{~h}_{4}(t), \\
v_{4}(t):=v_{f} \mathrm{~h}_{4}(t),
\end{gathered}
$$

and

$$
p_{4}(t):=\operatorname{sgn}\left(v_{m}\right) L \mathrm{~h}_{4}(t)
$$

where the function $\mathrm{h}_{4}(t)$ is defined similarly to that in (25), and the expression of $t_{5}$, which appears in (25), is furnished in Subsubsection 2.2.5.

Notice the similitude between this phase and the second phase of the basic version in Subsubsection 2.1.4.

\subsubsection{Pre-computation}

Let the adjusted minimum time to reach $v_{m}$ from $v_{i}$, which is $t_{a} \in \mathbb{R}_{>0}[s]$, and the adjusted minimum time to reach $v_{f}$ from $v_{m}$, which is $t_{d} \in \mathbb{R}_{>0}[s]$, be given by

$$
t_{a}:=\left(v_{m} / V_{d}\right) T_{a},
$$

and

$$
t_{d}:=\left(v_{m} / V_{d}\right) T_{d},
$$

and also the maximum (minimum) acceleration and deceleration be defined as

$$
a_{m}:=v_{m} / t_{a}
$$

and

$$
d_{m}:=-\left(v_{m} / t_{d}\right)
$$

The time constants $\tau_{11}$ through $\tau_{32}$ are defined as

$$
\begin{gathered}
\tau_{11}:=\left(\frac{v_{m}-v_{i}}{v_{m}}\right)\left(\frac{\theta_{a} t_{a}}{\xi_{a}-1}\right), \\
\tau_{12}:=\left(\frac{v_{m}-v_{i}}{v_{m}}\right)\left(1-\theta_{a}\right) t_{a}, \\
\tau_{31}:=\left(\frac{v_{m}-v_{f}}{v_{m}}\right)\left(\frac{\left(1-\theta_{d}\right) t_{d}}{\xi_{d}-1}\right),
\end{gathered}
$$

and

$$
\tau_{32}:=\left(\frac{v_{m}-v_{f}}{v_{m}}\right) \theta_{d} t_{d} .
$$

The auxiliary time instants $T_{i} \in \mathbb{R}_{>0}[\mathrm{~s}], i \in\{1,2,4$, 5 , where $T_{i}:=t_{i}^{-}$, are defined similarly to those in (39)-(40) and (42)-(43). Notice that $T_{3}$ has purposefully been skipped. It is separately treated ahead.

The auxiliary lengths $L_{11}, L_{12}, L_{2}, L_{31}, L_{32} \in \mathbb{R}_{\geqslant 0}$ $[\mathrm{m}]$ are defined as (notice the modulus function)

$$
\begin{gathered}
L_{11}:=\mid v_{i} T_{1}+a_{m} T_{1}^{2} / 2-a_{m} \tau_{11} T_{1} \\
+a_{m} \tau_{11}^{2}\left(1-\exp \left(-\xi_{a}\right)\right) \mid, \\
L_{12}:=\left|v_{m} T_{2}-\left(v_{m}-v_{i}\right)\left(1-\theta_{a}\right) \tau_{12}\left(1-\theta_{0}\right)\right|, \\
L_{31}:=\mid v_{m} T_{4}+d_{m} T_{4}^{2} / 2-d_{m} \tau_{31} T_{4} \\
+d_{m} \tau_{31}^{2}\left(1-\exp \left(-\xi_{d}\right)\right) \mid \\
L_{32}:=\left|v_{f} T_{5}+\left(v_{m}-v_{f}\right) \theta_{d} \tau_{32}\left(1-\theta_{0}\right)\right|,
\end{gathered}
$$

and

$$
L_{2}:=L-\left(L_{11}+L_{12}+L_{31}+L_{32}\right) .
$$

Finally, any robust, fast-execution numerical method can be employed to determine $v_{m}$ through the recursive use of (61)-(73), including the computation of $T_{1}, T_{2}$, $T_{4}$, and $T_{5}$, such that the three following conditions

$$
\begin{cases}L_{2} \geqslant L \min \left\{\epsilon_{L}, 0.1\right\}, & \text { and } \\ \left|v_{m}\right| \leqslant\left|V_{d}\right|, & \text { and } \\ \left|v_{i}\right|,\left|v_{f}\right| \leqslant\left|v_{m}\right| & \end{cases}
$$

are satisfied with the highest $\left|v_{m}\right|$. To begin with, $v_{m}=$ $V_{d}, v_{i}=V_{i}$, and $v_{f}=V_{f}$ are tested. If the conditions in (74) are not satisfied, then $\left|v_{m}\right|<\left|V_{d}\right|$ is used. Notice that it may also be needed to adjust $v_{i}$ and $v_{f}$ in order to ensure that $\left|v_{i}\right| \leqslant\left|v_{m}\right|$ and $\left|v_{f}\right| \leqslant\left|v_{m}\right|$, in case $\left|v_{m}\right|$ drops down to less than $\left|V_{i}\right|$ or $\left|V_{f}\right|$. 
Then, the positions $P_{i}, i \in\{1,2,3,4\}$, are respectively defined as

$$
\begin{gathered}
P_{1}:=\operatorname{sgn}\left(V_{d}\right) L_{11}, \\
P_{2}:=\operatorname{sgn}\left(V_{d}\right) L_{12}+P_{1}, \\
P_{3}:=\operatorname{sgn}\left(V_{d}\right) L_{2}+P_{2},
\end{gathered}
$$

and

$$
P_{4}:=\operatorname{sgn}\left(V_{d}\right) L_{31}+P_{3} .
$$

Lastly, $T_{3}$ is defined as

$$
T_{3}:=\left|L_{2} / v_{m}\right|
$$

and the function switching time instants $t_{i}, i \in\{1,2,3$, $4,5\}$, are consecutively defined, with respect to the initial instant $t_{0}$, in a similar fashion to those in (44).

\subsubsection{Simulation results}

See Subsection 4.2 for the results.

\subsection{Further features}

Both the basic and the advanced versions can be coded together, such that they can be somewhat interchangeable. Then, the use of the tuning parameters will dictate which version is the best in every case.

So far, both versions of the RM work in open-loop. However, the use of reference and state - either directly measured or estimated - feedback opens up new possibilities. For instance, the following can be listed:

- Hysteretic waiting function: by monitoring how closely an ROV tracks the synthesised position reference, it is possible to 'wait' for the ROV, for how long it is needed, in case the ROV is lagging behind such reference, until the difference is reduced to an acceptable value. Since the references are directly synthesised, it is possible to hold the value of the position reference without affecting the velocity reference. Such a measure has to be applied preferably during the second phase, because the acceleration reference is null during this case;

- Emergency stop function: if a collision against a stationary object, or against the seabed, is in the imminence of happening, for instance, it is a good idea to bring the ROV to a halt in the shortest time possible. Towards this end, it is possible to also code a slightly modified version of the third phase of the basic version, which is to be used in exceptional circumstances, such that both the acceleration and velocity references are quickly, although not instantly, zeroed, and the ROV at risk of collision is quickly kept safe in DP mode.

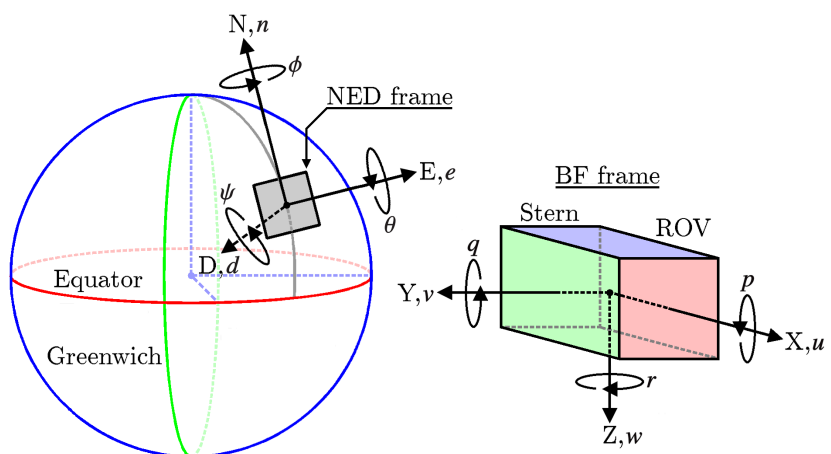

Figure 2: NED and BF reference frames.

\section{Path generation}

\subsection{Introduction}

Motion control applications concerning UUVs typically require the use of two reference frames simultaneously (Sørensen, 2013; Fossen, 2011; SNAME, 1950). The locally inertial North-East-Down (NED) reference frame, see Figure 2, serves as the reference for the following vector of desired position and attitude (heading angle)

$$
\boldsymbol{\eta}_{\boldsymbol{d}}(t):=\left[\begin{array}{llll}
n_{d}(t) & e_{d}(t) & d_{d}(t) & \psi_{d}(t)
\end{array}\right]^{T},
$$

where the elements $n_{d}(t), e_{d}(t)$, and $d_{d}(t)$ are respectively related to the $\mathrm{N}$-, E-, and D-axis, and $\psi_{d}(t)$ is the heading angle measured with respect to the $\mathrm{N}$-axis. The NED frame is typically used for locally flat Earth navigation. The (non-inertial) Body-Fixed (BF) reference frame, see Figure 2, serves as the reference for the vector of desired linear and angular velocities $\boldsymbol{\nu}_{\boldsymbol{d}}=$ $\left[u_{d}(t), v_{d}(t), w_{d}(t), r_{d}(t)\right]^{T}$, and likewise for the vector of desired linear and angular accelerations $\frac{\mathrm{d}}{\mathrm{dt}}\left[\boldsymbol{\nu}_{\boldsymbol{d}}(t)\right]=$ $\dot{\nu}_{d}=\left[\dot{u}_{d}(t), \dot{v}_{d}(t), \dot{w}_{d}(t), \dot{r}_{d}(t)\right]^{T}$, where the pairs $u_{d}(t)$ and $\dot{u}_{d}(t), v_{d}(t)$ and $\dot{v}_{d}(t), w_{d}(t)$ and $\dot{w}_{d}(t)$, and $r_{d}(t)=$ $\frac{\mathrm{d}}{\mathrm{dt}}\left[\psi_{d}(t)\right]$ and $\dot{r}_{d}(t)$ are respectively related to the motion in surge, sway, heave, and yaw. The origin of the $\mathrm{BF}$ frame is fixed at a convenient point of the UUV. The transformation matrix $\boldsymbol{J}\left(\psi_{d}(t)\right) \in \mathrm{SO}(4)$ (Special Orthogonal group of order 4 ), which is defined as

$$
\boldsymbol{J}\left(\psi_{d}(t)\right):=\left[\begin{array}{cccc}
\cos \left(\psi_{d}(t)\right) & -\sin \left(\psi_{d}(t)\right) & 0 & 0 \\
\sin \left(\psi_{d}(t)\right) & \cos \left(\psi_{d}(t)\right) & 0 & 0 \\
0 & 0 & 1 & 0 \\
0 & 0 & 0 & 1
\end{array}\right]
$$

transforms $\boldsymbol{\nu}_{\boldsymbol{d}}(t)$ into $\dot{\boldsymbol{\eta}}_{\boldsymbol{d}}(t)=\frac{\mathrm{d}}{\mathrm{dt}}\left[\boldsymbol{\eta}_{\boldsymbol{d}}(t)\right]$, such that

$$
\boldsymbol{\nu}_{\boldsymbol{d}}(t):=\boldsymbol{J}^{T}\left(\psi_{d}(t)\right) \dot{\boldsymbol{\eta}}_{\boldsymbol{d}}(t),
$$

and

$$
\dot{\boldsymbol{\nu}}_{\boldsymbol{d}}(t)=\dot{\boldsymbol{J}}^{T}\left(\psi_{d}(t)\right) \dot{\boldsymbol{\eta}}_{\boldsymbol{d}}(t)+\boldsymbol{J}^{T}\left(\psi_{d}(t)\right) \ddot{\boldsymbol{\eta}}_{\boldsymbol{d}}(t)
$$




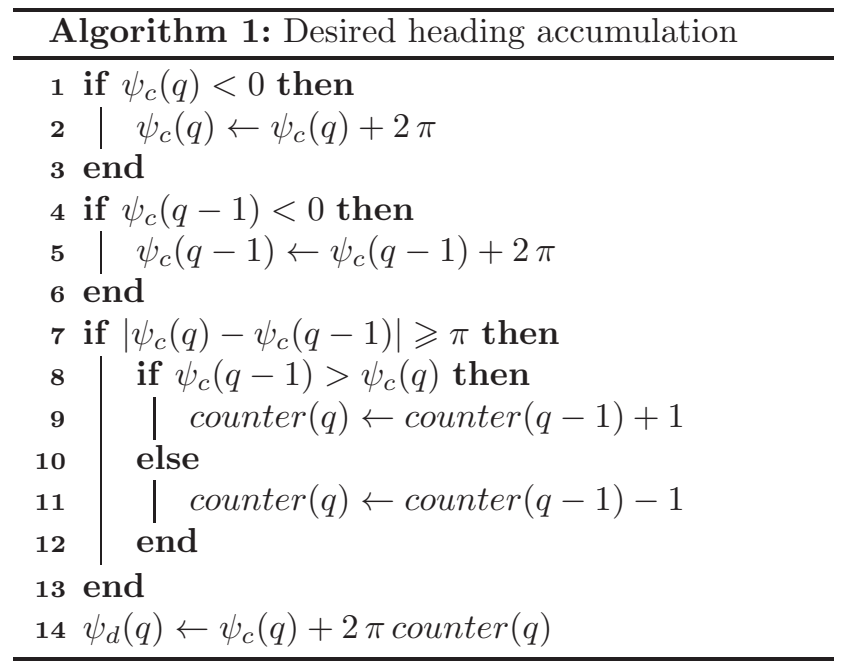

The desired heading angle $\psi_{d}(t)$ has to be accumulated along consecutive turns, e.g. through the use of Algorithm 1, to be in (80), for the sake of continuity beyond the natural supremum - either $2 \pi$ or $\pi$ [rad], depending on the considered range - , and the natural infimum - either 0 or $-\pi[\mathrm{rad}]$, depending on the considered range, i.e. to avoid step discontinuities at the aforementioned infimum and supremum.

The accumulated heading $\psi_{d}(q)$ that comes out from Algorithm 1, $q \in \mathbb{N} \backslash\{0\}$, when it comes to a discretetime implementation, is based on the two most recently computed values of the heading, $\psi_{c}(q)$ and $\psi_{c}(q-1)$, which have the same bounded range, and on the variable counter $\in \mathbb{Z}$, which accumulates the number of turns taken either Clockwise $(\mathrm{CW})$ (counter $\geqslant 0$ ), or Anticlockwise $(\mathrm{ACW})($ counter $<0)$. The variable is to be initialised as counter $(1)=$ counter $(0)=0$.

There are different ways and purposes of defining paths. This work is not concerned with the path planning problem, as mentioned before. It is therefore assumed herein that purposeful planar and spatial curvature- and torsion-continuous paths composed of rectilinear and curvilinear parts are previously defined, either through the use of proper path planning techniques, or directly based on the target applications. Curvature and torsion continuity imply that two connected parts share common centres of curvature and torsion at the join point. To illustrate the point of this section, paths which are comprised of straight lines connected through planar clothoids are used, without loss of generality. In fact, any planar curved line that is capable of satisfying the curvature continuity condition can be used instead. For instance, the Fermat's spiral (Lekkas, 2014; Lekkas et al., 2013), the single polar polynomial (Lai et al., 2007; Nelson, 1989), the cubic Bézier curve (Farouki, 2012; Seidel, 1993), and some
Pythagorean hodographs (Farouki, 2008, 1997; Farouki and Sakkalis, 1990), among others, are suitable alternatives.

\subsection{Straight lines}

Straight lines are fundamental building blocks of curvature- and torsion-continuous paths formed by rectilinear and curvilinear parts.

The length $L_{l} \in \mathbb{R}_{>0}[\mathrm{~m}]$ of a straight line is given by

$$
L_{l}:=\left\|\left(n_{l}^{1}, e_{l}^{1}, d_{l}^{1}\right)-\left(n_{l}^{0}, e_{l}^{0}, d_{l}^{0}\right)\right\|_{2},
$$

where the superscripts ${ }^{\circ} 0$, and ' 1 ' are not exponents in this case, but help to identify the start point $\left(n_{l}^{0}, e_{l}^{0}, d_{l}^{0}\right)$ and the endpoint $\left(n_{l}^{1}, e_{l}^{1}, d_{l}^{1}\right)$, whose coordinates in the NED frame are $n_{l}^{(\cdot)}, e_{l}^{(\cdot)}, d_{l}^{(\cdot)} \in \mathbb{R}[\mathrm{m}]$.

The elements of the points $\left(n_{l}\left(\varpi_{l}(t)\right), e_{l}\left(\varpi_{l}(t)\right)\right.$, $\left.d_{l}\left(\varpi_{l}(t)\right)\right)[\mathrm{m}]$, which describe the straight line in the NED frame, are given by

$$
\begin{aligned}
& n_{l}\left(\varpi_{l}(t)\right):=n_{l}^{0}+\varpi_{l}(t) L_{l} \cos \left(\alpha_{l}^{v}\right) \cos \left(\alpha_{l}^{h}\right), \\
& e_{l}\left(\varpi_{l}(t)\right):=e_{l}^{0}+\varpi_{l}(t) L_{l} \cos \left(\alpha_{l}^{v}\right) \sin \left(\alpha_{l}^{h}\right),
\end{aligned}
$$

and

$$
d_{l}\left(\varpi_{l}(t)\right):=d_{l}^{0}+\varpi_{l}(t) L_{l} \sin \left(\alpha_{l}^{v}\right),
$$

where $\varpi_{l}(t) \in \mathbb{R}_{\geqslant 0} \mid \varpi_{l}(t) \in[0,1] \forall t \in \mathbb{R}_{\geqslant 0}$ is the timedependent parameter that parameterises the straight line, $\alpha_{l}^{h} \in \mathbb{R} \mid \alpha_{l}^{h} \in(-\pi, \pi][\mathrm{rad}]$, which is a constant angle contained in the NE-plane that is measured from the N-axis, and $\alpha_{l}^{v} \in \mathbb{R} \mid \alpha_{l}^{v} \in[-\pi / 2, \pi / 2]$ [rad], which is a constant angle contained in a vertical plane that is measured from the NE-plane, are given by

$$
\alpha_{l}^{h}:=\operatorname{atan} 2\left(\left(e_{l}^{1}-e_{l}^{0}\right),\left(n_{l}^{1}-n_{l}^{0}\right)\right),
$$

and

$$
\alpha_{l}^{v}:=\tan ^{-1}\left(\frac{d_{l}^{1}-d_{l}^{0}}{\left\|\left(n_{l}^{1}, e_{l}^{1}\right)-\left(n_{l}^{0}, e_{l}^{0}\right)\right\|_{2}}\right) .
$$

The corresponding velocities $\dot{n}_{l}\left(\dot{\varpi}_{l}(t)\right), \dot{e}_{l}\left(\dot{\varpi}_{l}(t)\right)$, and $\dot{d}_{l}\left(\dot{\varpi}_{l}(t)\right)[\mathrm{m} / \mathrm{s}]$, and accelerations $\ddot{n}_{l}\left(\ddot{\varpi}_{l}(t)\right)$, $\ddot{e}_{l}\left(\ddot{\varpi}_{l}(t)\right)$, and $\ddot{d}_{l}\left(\ddot{\varpi}_{l}(t)\right)\left[\mathrm{m} / \mathrm{s}^{2}\right]$, are given by

$$
\begin{gathered}
\dot{n}_{l}\left(\dot{\varpi}_{l}(t)\right)=\dot{\varpi}_{l}(t) L_{l} \cos \left(\alpha_{l}^{v}\right) \cos \left(\alpha_{l}^{h}\right), \\
\dot{e}_{l}\left(\dot{\varpi}_{l}(t)\right)=\dot{\varpi}_{l}(t) L_{l} \cos \left(\alpha_{l}^{v}\right) \sin \left(\alpha_{l}^{h}\right), \\
\dot{d}_{l}\left(\dot{\varpi}_{l}(t)\right)=\dot{\varpi}_{l}(t) L_{l} \sin \left(\alpha_{l}^{v}\right), \\
\ddot{n}_{l}\left(\ddot{\varpi}_{l}(t)\right)=\ddot{\varpi}_{l}(t) L_{l} \cos \left(\alpha_{l}^{v}\right) \cos \left(\alpha_{l}^{h}\right), \\
\ddot{e}_{l}\left(\ddot{\varpi}_{l}(t)\right)=\ddot{\varpi}_{l}(t) L_{l} \cos \left(\alpha_{l}^{v}\right) \sin \left(\alpha_{l}^{h}\right),
\end{gathered}
$$

and

$$
\ddot{d}_{l}\left(\ddot{\varpi}_{l}(t)\right)=\ddot{\varpi}_{l}(t) L_{l} \sin \left(\alpha_{l}^{v}\right) .
$$




\subsection{Mirror-symmetric twin clothoids}

The clothoid, also known as the Euler spiral, or the Cornu spiral, is a plane curve whose curvature changes linearly with the arc length (Levien, 2008). Harary and Tal (2012) extended it to three dimensions, such that the torsion also changes linearly with the arc length. These characteristics can be explored for constructing curvature- and torsion-continuous paths, which are formed by straight lines connected through pairs of mirror-symmetric twin clothoids. Every pair of twin clothoids presents symmetry with respect to the line bisecting the (total) angle span. The (total) arc length can be exactly and easily computed based on the angle span. By knowing the arc length, the angle span, the rotation direction, and the parity, it is possible to determine the endpoint of the pair of twin clothoids, through the accurate computation of the midpoint, based on the start point. Only planar clothoids are considered here.

The arc length $L_{c} \in \mathbb{R}_{>0}[\mathrm{~m}]$ of a pair of mirror-symmetric twin clothoids that winds from the start point, at which the curvature is null, to the midpoint, at which the curvature is maximum, and thence unwinds to the endpoint, at which the curvature is equal to zero again, is given by

$$
L_{c}:=2 \theta_{c} / \kappa_{c}^{\max },
$$

where $\theta_{c} \in \mathbb{R}_{>0} \mid \theta_{c} \in(0, \pi][\mathrm{rad}]$ is the angle span, and $\kappa_{c}^{\max }=\left(1 / r_{c}^{\min }\right) \in \mathbb{R}_{>0}\left[\mathrm{~m}^{-1}\right]$ is the maximum admissible curvature, where $r_{c}^{\text {min }} \in \mathbb{R}_{>0}[\mathrm{~m}]$ is the minimum radius of curvature, which occurs at the midpoint, i.e. at the point where $L_{c} / 2$ and $\theta_{c} / 2$. It is worth knowing that the arc length ratio $L_{c} / L_{r}$, where $L_{r} \in \mathbb{R}_{>0}[\mathrm{~m}]$ is the length of a virtual arc of circumference - shortest arc — whose start point and endpoint coincide with those of the pair of twin clothoids, is closely approximated by the curve depicted in Figure 3. Notice that $L_{c}$ becomes more than $10 \%$ longer than $L_{r}$ only for $\theta_{c}$ $>105.4^{\circ}$. The radius ratio $r_{r} / r_{c}^{\mathrm{min}}$, where $r_{r} \in \mathbb{R}_{>0}[\mathrm{~m}]$ is the radius of the virtual arc of circumference whose length is equal to $L_{r}$, is closely approximated by the curve depicted in Figure 3. Notice that for $\theta_{c}<20^{\circ}$, $\kappa_{c}^{\max }$ is approximately twice that of the circumference.

The elements of the points $\left(n_{c}\left(\varpi_{c}(t)\right), e_{c}\left(\varpi_{c}(t)\right)\right)$ [m], which describe the pair of twin clothoids in the NE-plane, and the tangent angles $\alpha_{c}\left(\varpi_{c}(t)\right)[\mathrm{rad}]$ at the points, are given by

$$
\begin{aligned}
n_{c}\left(\varpi_{c}(t)\right):= & n_{c}^{0}+n_{c}^{1}\left(\varpi_{c}(t)\right)\left(\mathrm{H}\left(\varpi_{c}(t)\right)-\mathrm{H}\left(\varpi_{c}(t)-0.5\right)\right) \\
& +n_{c}^{2}\left(\varpi_{c}(t)\right)\left(\mathrm{H}\left(\varpi_{c}(t)-0.5\right)-\mathrm{H}\left(\varpi_{c}(t)-1\right)\right),
\end{aligned}
$$$$
e_{c}\left(\varpi_{c}(t)\right):=e_{c}^{0}+e_{c}^{1}\left(\varpi_{c}(t)\right)\left(\mathrm{H}\left(\varpi_{c}(t)\right)-\mathrm{H}\left(\varpi_{c}(t)-0.5\right)\right)
$$

$$
+e_{c}^{2}\left(\varpi_{c}(t)\right)\left(\mathrm{H}\left(\varpi_{c}(t)-0.5\right)-\mathrm{H}\left(\varpi_{c}(t)-1\right)\right),
$$

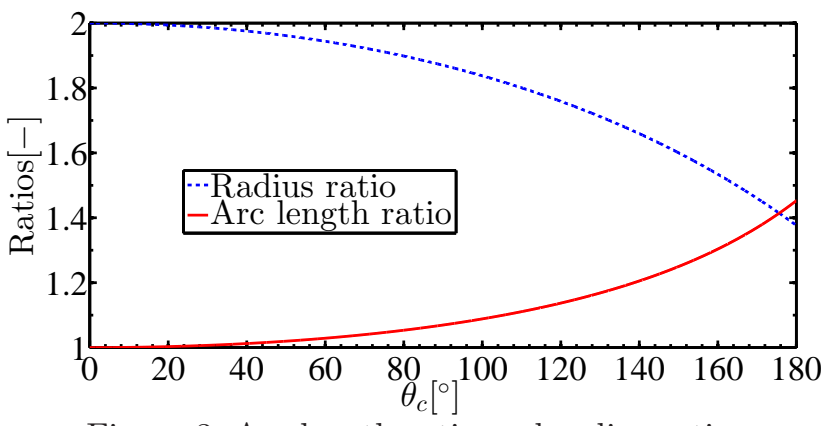

Figure 3: Arc length ratio and radius ratio.

and

$\alpha_{c}\left(\varpi_{c}(t)\right):=\alpha_{c}^{0}+\alpha_{c}^{1}\left(\varpi_{c}(t)\right)\left(\mathrm{H}\left(\varpi_{c}(t)\right)-\mathrm{H}\left(\varpi_{c}(t)-0.5\right)\right)$

$$
+\alpha_{c}^{2}\left(\varpi_{c}(t)\right)\left(\mathrm{H}\left(\varpi_{c}(t)-0.5\right)-\mathrm{H}\left(\varpi_{c}(t)-1\right)\right),
$$

where the superscripts ${ }^{~} 0$, , '1, and '2, are not exponents, $\varpi_{c}(t) \in \mathbb{R}_{\geqslant 0} \mid \varpi_{c}(t) \in[0,1] \forall t \in \mathbb{R}_{\geqslant 0}$ is the time-dependent parameter that parameterises the pair of twin clothoids, $\left(n_{c}^{0}, e_{c}^{0}\right)$ is the start point, whose coordinates are $n_{c}^{0} \in \mathbb{R}[\mathrm{m}]$ (north) and $e_{c}^{0} \in \mathbb{R}[\mathrm{m}]$ (east), $\alpha_{c}^{0} \in$ $\mathbb{R} \mid \alpha_{c}^{0} \in(-\pi, \pi][\mathrm{rad}]$ is the tangent angle at the point $\left(n_{c}^{0}, e_{c}^{0}\right)$, and $\mathrm{H}(\cdot)$ is the unit step function.

The parameter $\varpi_{c}(t)$ ranges from zero to 0.5 along the first clothoid arc of the pair of twin clothoids, as indicated in (97)-(99). The terms $n_{c}^{1}\left(\varpi_{c}(t)\right)[\mathrm{m}]$ and $e_{c}^{1}\left(\varpi_{c}(t)\right)[\mathrm{m}]$ are given by

$$
\left[\begin{array}{c}
n_{c}^{1}\left(\varpi_{c}(t)\right) \\
e_{c}^{1}\left(\varpi_{c}(t)\right)
\end{array}\right]:=\left[\begin{array}{cc}
\cos \left(\alpha_{c}^{0}\right) & -\sin \left(\alpha_{c}^{0}\right) \\
\sin \left(\alpha_{c}^{0}\right) & \cos \left(\alpha_{c}^{0}\right)
\end{array}\right]\left[\begin{array}{c}
c_{c}^{\prime}\left(\varpi_{c}(t)\right) \\
s_{c}^{\prime}\left(\varpi_{c}(t)\right)
\end{array}\right],
$$

where $c_{c}^{\prime}\left(\varpi_{c}(t)\right)$ and $s_{c}^{\prime}\left(\varpi_{c}(t)\right)$ are given by the following absolutely convergent power series

$$
c_{c}^{\prime}\left(\varpi_{c}(t)\right):=\frac{1}{\kappa_{c}^{\max }} \sum_{k=0}^{\infty} \frac{(-1)^{k} \theta_{c}^{2 k+1}\left(2 \varpi_{c}(t)\right)^{4 k+1}}{(2 k) !(4 k+1) 2^{2 k}},
$$

and

$$
s_{c}^{\prime}\left(\varpi_{c}(t)\right):=\frac{\lambda_{c}}{\kappa_{c}^{\max }} \sum_{k=0}^{\infty} \frac{(-1)^{k} \theta_{c}^{2 k+2}\left(2 \varpi_{c}(t)\right)^{4 k+3}}{(2 k+1) !(4 k+3) 2^{2 k+1}},
$$

where $\lambda_{c} \in \mathbb{Z} \mid \lambda_{c} \in\{-1,1\}$ denotes the rotation direction developed from the point $\left(n_{c}^{0}, e_{c}^{0}\right)$, i.e. $\lambda_{c}=1$ assigns the CW direction, whereas $\lambda_{c}=-1$ assigns the ACW direction.

The tangent angles $\alpha_{c}^{1}\left(\varpi_{c}(t)\right)$ [rad] are given by

$$
\alpha_{c}^{1}\left(\varpi_{c}(t)\right):=2 \lambda_{c} \theta_{c} \varpi_{c}^{2}(t)
$$

The parameter $\varpi_{c}(t)$ ranges from 0.5 to 1 along the second clothoid arc of the pair of twin clothoids, as 
indicated in (97)-(99). The terms $n_{c}^{2}\left(\varpi_{c}(t)\right)[\mathrm{m}]$ and $e_{c}^{2}\left(\varpi_{c}(t)\right)[\mathrm{m}]$ are given by

$$
\left[\begin{array}{c}
n_{c}^{2}\left(\varpi_{c}(t)\right) \\
e_{c}^{2}\left(\varpi_{c}(t)\right)
\end{array}\right]:=\left[\begin{array}{cc}
\cos \left(\alpha_{c}^{0}\right) & -\sin \left(\alpha_{c}^{0}\right) \\
\sin \left(\alpha_{c}^{0}\right) & \cos \left(\alpha_{c}^{0}\right)
\end{array}\right]\left[\begin{array}{c}
c_{c}^{\prime \prime}\left(\varpi_{c}(t)\right) \\
s_{c}^{\prime \prime}\left(\varpi_{c}(t)\right)
\end{array}\right],
$$

where $c_{c}^{\prime \prime}\left(\varpi_{c}(t)\right)$ and $s_{c}^{\prime \prime}\left(\varpi_{c}(t)\right)$ are given by

$$
\begin{aligned}
& {\left[\begin{array}{c}
c_{c}^{\prime \prime}\left(\varpi_{c}(t)\right) \\
s_{c}^{\prime \prime}\left(\varpi_{c}(t)\right)
\end{array}\right]:=\left[\begin{array}{l}
c_{c}^{\prime}(0.5) \\
s_{c}^{\prime}(0.5)
\end{array}\right]+} \\
& \quad\left[\begin{array}{rr}
\cos \left(\chi_{c}\right) & -\rho_{c} \sin \left(\chi_{c}\right) \\
-\sin \left(\chi_{c}\right) & -\rho_{c} \cos \left(\chi_{c}\right)
\end{array}\right]\left[\begin{array}{c}
c_{c}^{\prime \prime \prime}\left(\varpi_{c}(t)\right)-c_{c}^{\prime}(0.5) \\
s_{c}^{\prime \prime \prime}\left(\varpi_{c}(t)\right)-s_{c}^{\prime}(0.5)
\end{array}\right],
\end{aligned}
$$

where $\rho_{c} \in \mathbb{Z} \mid \rho_{c} \in\{-1,1\}$ denotes the parity, i.e. $\rho_{c}=$ 1 assigns the even parity, which results in a symmetric pair of twin clothoids, whereas $\rho_{c}=-1$ assigns the odd parity, which results in an antisymmetric pair of twin clothoids. The latter pair presents a curvature discontinuity at the midpoint, due to an instantaneous change of the centre of curvature at the midpoint, which causes such pair of clothoids to degenerate into just tangentcontinuous $\left(G^{1}\right)$. The argument $\chi_{c}$ is defined as

$$
\chi_{c}:=\lambda_{c}\left(\pi-\left(1+\rho_{c}\right) \theta_{c} / 2\right),
$$

and $c_{c}^{\prime \prime \prime}\left(\varpi_{c}(t)\right)$ and $s_{c}^{\prime \prime \prime}\left(\varpi_{c}(t)\right)$ are given by

$c_{c}^{\prime \prime \prime}\left(\varpi_{c}(t)\right):=\frac{1}{\kappa_{c}^{\max }} \sum_{k=0}^{\infty} \frac{(-1)^{k} \theta_{c}^{2 k+1}\left(2\left(1-\varpi_{c}(t)\right)\right)^{4 k+1}}{(2 k) !(4 k+1) 2^{2 k}}$,

and

$$
s_{c}^{\prime \prime \prime}\left(\varpi_{c}(t)\right):=\frac{\lambda_{c}}{\kappa_{c}^{\max }} \sum_{k=0}^{\infty} \frac{(-1)^{k} \theta_{c}^{2 k+2}\left(2\left(1-\varpi_{c}(t)\right)\right)^{4 k+3}}{(2 k+1) !(4 k+3) 2^{2 k+1}} .
$$

The tangent angles $\alpha_{c}^{2}\left(\varpi_{c}(t)\right)$ [rad] are given by

$$
\alpha_{c}^{2}\left(\varpi_{c}(t)\right):=\left(\lambda_{c} \theta_{c} / 2\right)\left(1+\rho_{c}\left(1-4\left(1-\varpi_{c}(t)\right)^{2}\right)\right) .
$$

The linear and angular velocities $\dot{n}_{c}\left(\varpi_{c}(t), \dot{\varpi}_{c}(t)\right)$ $[\mathrm{m} / \mathrm{s}], \quad \dot{e}_{c}\left(\varpi_{c}(t), \dot{\varpi}_{c}(t)\right)[\mathrm{m} / \mathrm{s}], \quad$ and $\quad \dot{\alpha}_{c}\left(\varpi_{c}(t), \dot{\varpi}_{c}(t)\right)$ $[\mathrm{rad} / \mathrm{s}]$ along the pair of twin clothoids are given by

$$
\begin{aligned}
& \dot{n}_{c}\left(\varpi_{c}(t), \dot{\varpi}_{c}(t)\right)=\frac{\mathrm{d}}{\mathrm{dt}}\left[n_{c}\left(\varpi_{c}(t)\right)\right], \\
& \dot{e}_{c}\left(\varpi_{c}(t), \dot{\varpi}_{c}(t)\right)=\frac{\mathrm{d}}{\mathrm{dt}}\left[e_{c}\left(\varpi_{c}(t)\right)\right],
\end{aligned}
$$

and

$$
\dot{\alpha}_{c}\left(\varpi_{c}(t), \dot{\varpi}_{c}(t)\right)=\frac{\mathrm{d}}{\mathrm{dt}}\left[\alpha_{c}\left(\varpi_{c}(t)\right)\right],
$$

and the corresponding linear and angular accelerations $\ddot{n}_{c}\left(\varpi_{c}(t), \dot{\varpi}_{c}(t), \ddot{\varpi}_{c}(t)\right)\left[\mathrm{m} / \mathrm{s}^{2}\right], \quad \ddot{e}_{c}\left(\varpi_{c}(t), \dot{\varpi}_{c}(t), \ddot{\varpi}_{c}(t)\right)$ $\left[\mathrm{m} / \mathrm{s}^{2}\right]$, and $\ddot{\alpha}_{c}\left(\varpi_{c}(t), \dot{\varpi}_{c}(t), \ddot{\varpi}_{c}(t)\right)\left[\mathrm{rad} / \mathrm{s}^{2}\right]$ are given by

$$
\ddot{n}_{c}\left(\varpi_{c}(t), \dot{\varpi}_{c}(t), \ddot{\varpi}_{c}(t)\right)=\frac{\mathrm{d}^{2}}{\mathrm{dt}^{2}}\left[n_{c}\left(\varpi_{c}(t)\right)\right],
$$

$$
\ddot{e}_{c}\left(\varpi_{c}(t), \dot{\varpi}_{c}(t), \ddot{\varpi}_{c}(t)\right)=\frac{\mathrm{d}^{2}}{\mathrm{dt}^{2}}\left[e_{c}\left(\varpi_{c}(t)\right)\right],
$$

and

$$
\ddot{\alpha}_{c}\left(\varpi_{c}(t), \dot{\varpi}_{c}(t), \ddot{\varpi}_{c}(t)\right)=\frac{\mathrm{d}^{2}}{\mathrm{dt}^{2}}\left[\alpha_{c}\left(\varpi_{c}(t)\right)\right],
$$

where the derivatives with respect to time in (110)(111) and (113)-(114) are obtained with the help of

$$
\begin{aligned}
\frac{\mathrm{d}^{n}}{\mathrm{dt}^{n}}\left[\phi_{c}\left(\varpi_{c}(t)\right)\right] & =\frac{\mathrm{d}^{n}}{\mathrm{dt}^{n}}\left[\zeta \sum_{k=0}^{\infty} \frac{\mathbb{N u m}\left(k, \varpi_{c}(t)\right)}{\operatorname{Den}(k)}\right] \\
& =\zeta \sum_{k=0}^{\infty} \frac{\mathrm{d}^{n}}{\mathrm{dt}^{n}}\left[\frac{\mathbb{N u m}\left(k, \varpi_{c}(t)\right)}{\operatorname{Den}(k)}\right],
\end{aligned}
$$

where $\phi_{c}\left(\varpi_{c}(t)\right)$ is a dummy function that represents $c_{c}^{\prime}\left(\varpi_{c}(t)\right), \quad s_{c}^{\prime}\left(\varpi_{c}(t)\right), \quad c_{c}^{\prime \prime \prime}\left(\varpi_{c}(t)\right), \quad$ and $s_{c}^{\prime \prime \prime}\left(\varpi_{c}(t)\right)$, in (101), (102), (107), and (108), $\zeta$ is a dummy constant that represents either $1 / \kappa_{c}^{\max }$ or $\lambda_{c} / \kappa_{c}^{\max }$, and $\operatorname{Num}\left(k, \varpi_{c}(t)\right)$ and $\mathbb{D e n}(k)$ respectively stand for the numerator and the denominator of $\phi_{c}\left(\varpi_{c}(t)\right)$.

Based on (110), (111), (113), and (114), the curvature is given by

$$
\kappa_{c}\left(\varpi_{c}(t)\right)=\frac{\left|\dot{n}_{c}(\cdot) \ddot{e}_{c}(\cdot)-\dot{e}_{c}(\cdot) \ddot{n}_{c}(\cdot)\right|}{\sqrt{\left(\left(\dot{n}_{c}(\cdot)\right)^{2}+\left(\dot{e}_{c}(\cdot)\right)^{2}\right)^{3}}} .
$$

Remark: The power series (101), (102), (107), and (108) are adapted from Råde and Westergren (2004). The magnitude of the terms in the sums to infinity (101), (102), (107), (108), and (116) decreases rapidly as $k$ increases. The use of only a few terms, e.g. $k \in$ $\{0,1, \ldots, 12\}$, yields very close approximations for the sums in practice. Exploring this fact leads to expedited, yet accurate, computations of those summands.

\subsection{Path generation}

There are essentially two possibilities of combining straight lines and pairs of twin clothoids - or other suitable types of curved lines - to form paths. The first possibility consists of using the pairs of twin clothoids as approximating curves with specified maximum curvatures, thereby resulting in paths which do not pass through all the waypoints which sketch out the paths. There are typically as many pairs of twin clothoids as the total number of waypoints minus two. Figure 4 shows an example in which all clothoids have the same maximum curvature. The second possibility consists of using the pairs of twin clothoids as interpolating curves with specified maximum curvatures, thereby resulting in paths which do pass through all the waypoints which sketch out the paths. Figure 5 shows an example in which all clothoids have the same maximum 


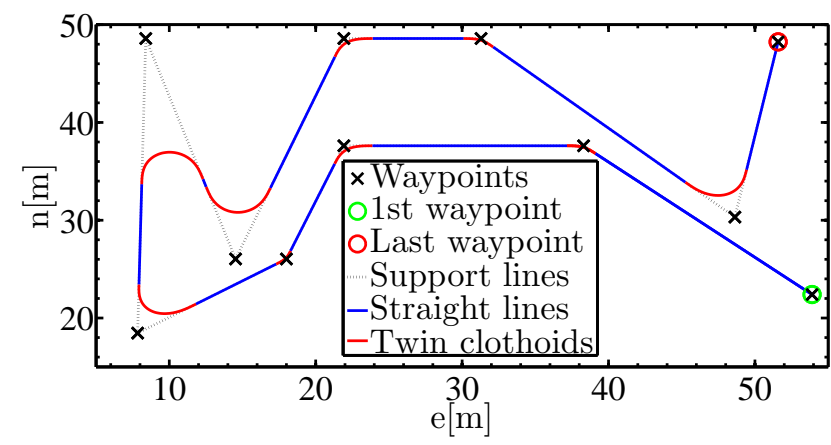

Figure 4: Example of path using approximating curves.

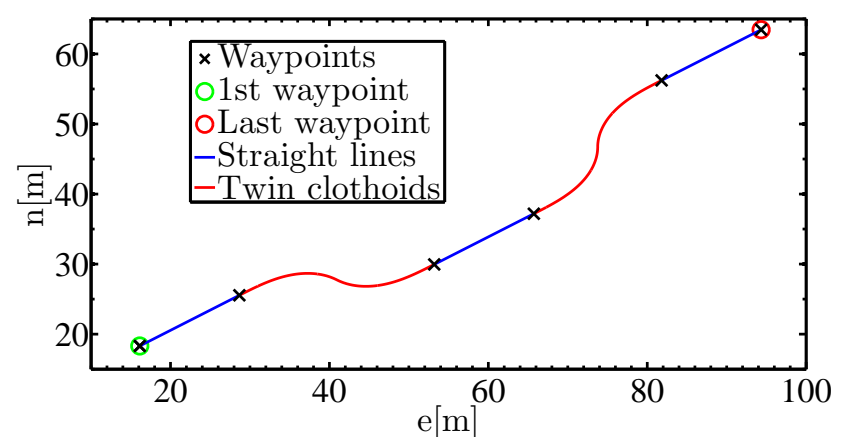

Figure 5: Example of path using interpolating curves.

curvature. Such a kind of path, which encompasses two successive lane changes, is useful, among other things, to avoid collisions.

The path in Figure 5 is based on antisymmetric pairs of twin clothoids — odd parity $\left(\rho_{c}=-1\right)$ - , which render the path only tangent-continuous $\left(G^{1}\right)$, due to the inherent properties of the clothoids. Notice that this fact has nothing to do with the curve interpolation. Alternatives to circumvent the curvature discontinuity problem are: i) to replace each antisymmetric pair of twin clothoids by two symmetric pairs of twin clothoids linked by a short straight line; or ii) to replaced them with curved lines which yield curvature-continuous lane change paths, e.g. the curve reported in Nelson (1989); or iii) to move along the path in such a manner that the velocity and the acceleration are both null at the join point of both clothoids of the antisymmetric pair.

The total path length $L_{p} \in \mathbb{R}_{>0}[\mathrm{~m}]$ is given by

$$
L_{p}:=\sum_{i=1}^{N} L_{i}
$$

where $L_{i} \in \mathbb{R}_{>0}[\mathrm{~m}]$ denotes the length of the $i$-th part of the path, which is formed by $N$ juxtaposed parts in total. In essence, the path length can be mapped, from the start point to the endpoint, as a strictly monotonically increasing one-to-one function of the parameter $\varpi_{p}(t) \in \mathbb{R}_{\geqslant 0} \mid \varpi_{p}(t) \in[0,1] \forall t \in \mathbb{R}_{\geqslant 0}$. Likewise, the length of the $i$-th part of the path can be mapped as a function of the parameter $\varpi_{i}(t) \in \mathbb{R}_{\geqslant 0} \mid \varpi_{i}(t) \in[0,1]$ $\forall t \in \mathbb{R}_{\geqslant 0}$. Then, either $\varpi_{l}(t)=\varpi_{i}(t), \dot{\varpi}_{l}(t)=\dot{\varpi}_{i}(t)$, and $\ddot{\varpi}_{l}(t)=\ddot{\varpi}_{i}(t)$, or $\varpi_{c}(t)=\varpi_{i}(t), \dot{\varpi}_{c}(t)=\dot{\varpi}_{i}(t)$, and $\ddot{\varpi}_{c}(t)=\ddot{\varpi}_{i}(t), i \in\{1,2, \ldots, N\}$, depending on the type of the line - rectilinear or curvilinear, respectively —, see Subsections 3.2-3.3.

The parameter $\varpi_{p}(t)$, and its derivatives with respect to time $\dot{\varpi}_{p}(t)$ and $\ddot{\varpi}_{p}(t)$, are defined as

$$
\begin{gathered}
\varpi_{p}(t)=\varpi_{p}(p(t)):=|p(t)| / L_{p}, \\
\dot{\varpi}_{p}(t)=\dot{\varpi}_{p}(v(t), p(t)):=\operatorname{sgn}(p(t)) v(t) / L_{p},
\end{gathered}
$$

and

$$
\ddot{\varpi}_{p}(t)=\ddot{\varpi}_{p}(a(t), p(t)):=\operatorname{sgn}(p(t)) a(t) / L_{p},
$$

where $a(t), v(t)$, and $p(t)$ come respectively from (1)(3). They can be generated by either the basic version of the RM that is described in Subsection 2.1, or an advanced version of it, as described in Subsection 2.2. The relation $|p(t)| \equiv \operatorname{sgn}(p(t)) p(t) \Leftrightarrow p(t) \in \mathbb{R} \backslash\{0\}$ is utilised to determine the derivatives with respect to time in (120)-(121). Consequently, the $N$ parameters $\varpi_{i}(t), i \in\{1,2, \ldots, N\}$, and their derivatives with respect to time $\dot{\varpi}_{i}(t)$ and $\ddot{\varpi}_{i}(t)$, are defined as

$$
\begin{gathered}
\varpi_{i}(t):=\frac{L_{p}}{L_{i}}\left(\varpi_{p}(t)-\sum_{j=1}^{i-1} \frac{L_{j}}{L_{p}}\right) \mathrm{h}_{i}\left(\varpi_{p}(t)\right), \\
\dot{\varpi}_{i}(t):=\left(L_{p} / L_{i}\right) \dot{\varpi}_{p}(t) \mathrm{h}_{i}\left(\varpi_{p}(t)\right),
\end{gathered}
$$

and

$$
\ddot{\varpi}_{i}(t):=\left(L_{p} / L_{i}\right) \ddot{\varpi}_{p}(t) \mathrm{h}_{i}\left(\varpi_{p}(t)\right),
$$

where $L_{j}=L_{i} \Leftrightarrow j=i$. The characteristic functions $\mathrm{h}_{i}\left(\varpi_{p}(t)\right)$ of the first $N-1$ (half-closed) subintervals of $\varpi_{p}(t)$, i.e. for $i \in\{1,2, \ldots, N-1\}$, are defined as

$$
\begin{aligned}
\mathrm{h}_{i}\left(\varpi_{p}(t)\right):= & \mathrm{H}\left(\frac{L_{p}}{L_{i}}\left(\varpi_{p}(t)-\sum_{j=1}^{i-1} \frac{L_{j}}{L_{p}}\right)\right) \\
& -\mathrm{H}\left(\frac{L_{p}}{L_{i+1}}\left(\varpi_{p}(t)-\sum_{j=1}^{i} \frac{L_{j}}{L_{p}}\right)\right),
\end{aligned}
$$

where $\mathrm{H}(\cdot)$ denotes the unit step function, whereas the characteristic function of the $N$-th (closed) subinterval of $\varpi_{p}(t)$, i.e. for $i=N$, is defined as

$$
\mathrm{h}_{i}\left(\varpi_{p}(t)\right):=\mathrm{H}\left(\frac{L_{p}}{L_{i}}\left(\varpi_{p}(t)-\sum_{j=1}^{i-1} \frac{L_{j}}{L_{p}}\right)\right) .
$$

\subsection{Simulation results}

See Subsection 4.3 for the results. 


\section{Selected results}

\subsection{Reference model: basic version: full-scale sea trials}

This subsection presents selected results from full-scale sea trials in which the ROV Minerva, see Appendix B, was operated in Trondheimsfjorden, Norway, in October, 2011. The four DoF MCS used to carry out the experiments was that reported in Sørensen et al. (2012), with the guidance subsystem running the algorithm of the basic version of the RM reported in Fernandes et al. (2012). The MCS was implemented on a compact Reconfigurable Input/Output (cRIO) module, and programmed via LabVIEW ${ }^{\circledR}$ for Microsoft Windows, both by National Instruments. It ran at the constant sampling frequency of $5 \mathrm{~Hz}$. The tuning parameters of the RM were $L=50 \mathrm{~m}, V_{d}=0.3 \mathrm{~m} / \mathrm{s}, T_{a}=20 \mathrm{~s}, T_{d}=20 \mathrm{~s}$, $\epsilon_{L}=0.05, \theta_{a}=0.8, \theta_{d}=0.2$, and $\theta_{0}=\exp (-11 . \overline{1})$, see Table 1 for details on them.

Figure 6 depicts a $50 \mathrm{~m}$-long surge motion along a straight line heading northwards from the local origin of the NED reference frame. This implies that $\psi_{d}(t)=$ $0^{\circ} \forall t \in \mathbb{R}_{\geqslant 0}$, whereupon $\boldsymbol{J}\left(\psi_{d}(t)\right)=\boldsymbol{I}$ in (81), where $\boldsymbol{I} \in \mathbb{R}^{4 \times 4}$ is an identity matrix, $\boldsymbol{\eta}_{\boldsymbol{d}}=[p(t), 0,0,0]^{T}$, $\boldsymbol{\nu}_{\boldsymbol{d}}=[v(t), 0,0,0]^{T}$, and $\dot{\boldsymbol{\nu}}_{\boldsymbol{d}}=[a(t), 0,0,0]^{T}$, in $(80)-$ (83). The maximum absolute (spatial) position error is $<0.5 \mathrm{~m}$, with maximum absolute depth error $<0.1 \mathrm{~m}$. The maximum absolute heading error is $<4^{\circ}$. Figure 7 depicts the corresponding velocities along the path. The measured and the estimated velocities remained close to the references all the time. Figure 8 depicts the commanded rotations of the propellers. The curves of the starboard and port thrusters are nearly flat, somewhat mimicking the shape of the reference surge velocity $u_{d}(t)$. The curve of the lateral thruster oscillates gently in order to maintain the desired heading and position, compensating for the motion disturbances. The curve of both vertical thrusters is practically flat all the time.

Fernandes et al. (2012) also report a simple performance comparison experiment concerning a single surge motion being guided by either the proposed RM and an FBRM (Fossen, 2011), where the proposed RM outperforms the FBRM, as expected.

\subsection{Reference model: advanced version: simulation results}

This subsection presents simulation results regarding the advanced version of the RM described in Subsection 2.2. The RM is studied in isolation, for the sake of clarity. References regarding a single DoF motion were synthesised. The simulation was based on recursive use

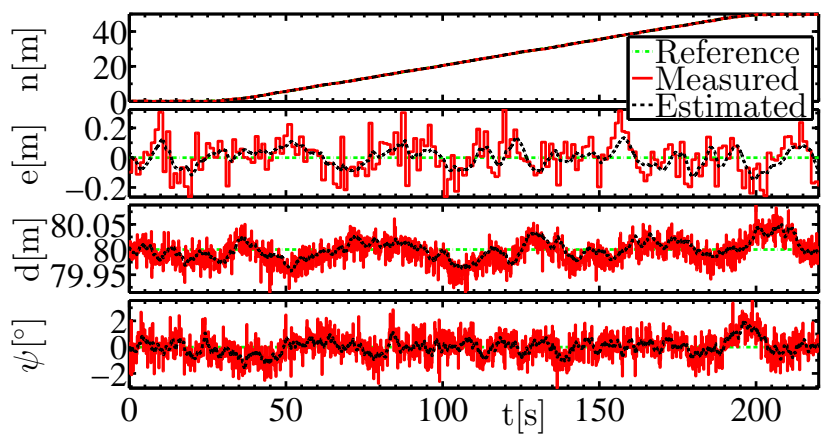

Figure 6: Position and heading (NED frame).

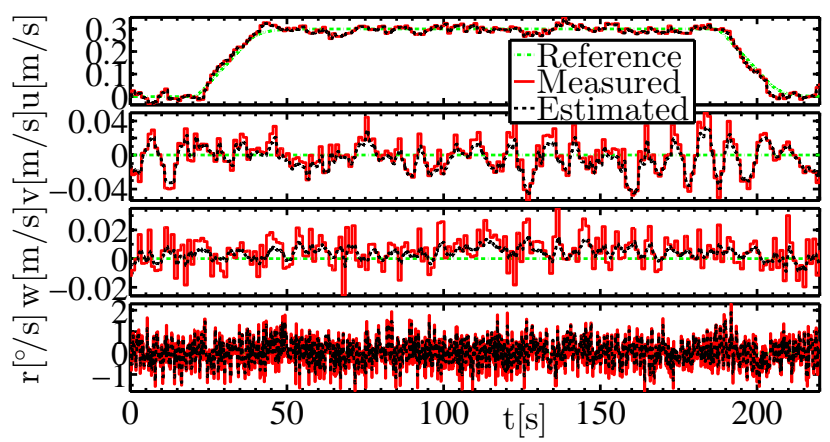

Figure 7: Linear and angular velocities (BF frame).

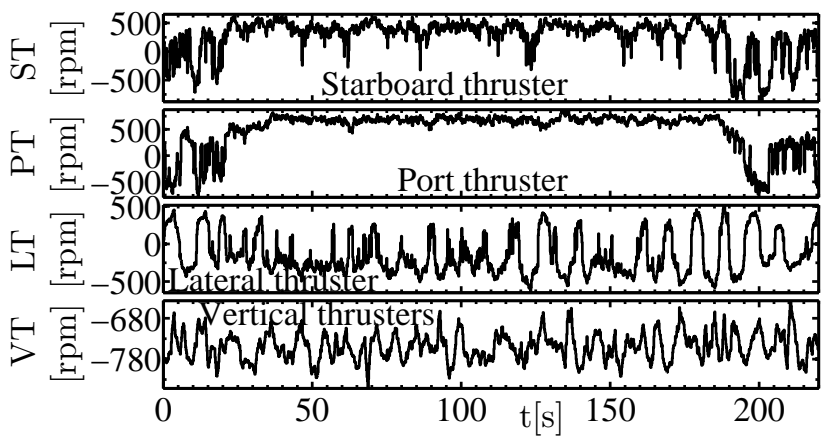

Figure 8: Commanded propeller rotations.

of the RM thrice. Different features of the RM were explored every time it was run. The constant sampling frequency was $6 . \overline{6} \mathrm{~Hz}$. The tuning parameters are collected in Table 2. See Table 1 for details about them.

The results are concentrated in Figure 9. The 250 mlong motion starts and ends at rest. It is worthwhile to realise that $v_{m}=V_{d}, v_{i}=V_{i}$, and $v_{f}=V_{f}$ in all cases. The vertical dashed red lines separate each case, which begin at $15 \mathrm{~s}, \approx 78 \mathrm{~s}$, and $\approx 128 \mathrm{~s}$, respectively. The latter ends at $\approx 224 \mathrm{~s}$. In the first case, the fourth phase is skipped, since the desired final velocity is not zero. In the second case, the first, third, and fourth phases are skipped, because the cruise velocity is held throughout. Finally, in the third case, all phases are executed. In particular, the fourth phase is executed due to the fact that the desired final velocity is equal to zero. 
Table 2: Tuning parameters of the RM

\begin{tabular}{lcccl}
\hline Parameter & $1 \mathrm{st}$ & 2nd & 3rd & Unit \\
\hline$L$ & 100 & 50 & 100 & $\mathrm{~m}$ \\
$V_{d}$ & 2 & 1 & 1.5 & $\mathrm{~m} / \mathrm{s}$ \\
$V_{i}$ & 0 & 1 & 1 & $\mathrm{~m} / \mathrm{s}$ \\
$V_{f}$ & 1 & 1 & 0 & $\mathrm{~m} / \mathrm{s}$ \\
$T_{a}$ & 10 & 10 & 10 & $\mathrm{~s}$ \\
$T_{d}$ & 10 & 10 & 10 & $\mathrm{~s}$ \\
$\epsilon_{L}$ & 0.05 & 0.05 & 0.05 & - \\
$\theta_{a}$ & 0.8 & 0.8 & 0.8 & - \\
$\theta_{d}$ & 0.2 & 0.2 & 0.2 & - \\
$\theta_{0}$ & $\exp (-13)$ & $\exp (-13)$ & $\exp (-13)$ & - \\
\hline
\end{tabular}

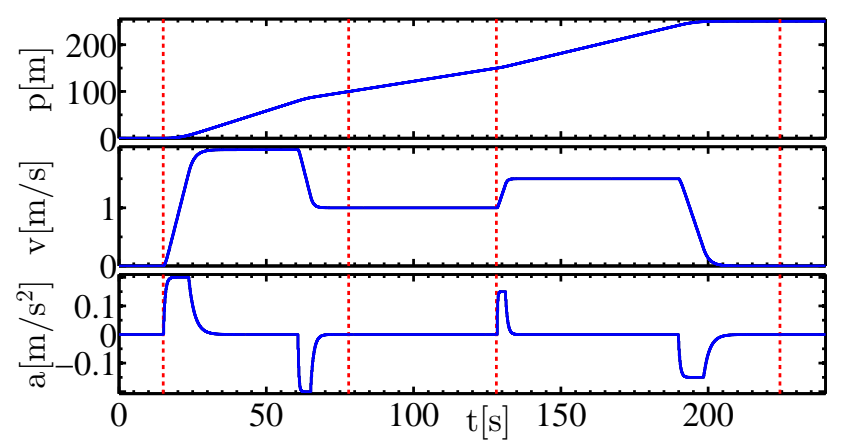

Figure 9: References synthesised by the advanced version of the RM.

\subsection{Path generation: simulation results}

This subsection presents simulation results based on the ROV Minerva, see Appendix B, regarding the path generation scheme described in Section 3. The four DoF MCS used to carry out the simulation was that reported in Fernandes et al. (2015), with the guidance subsystem built upon the just mentioned path generation scheme. The simulation was based on recursive use of the advanced version of the RM that is described in Subsection 2.2. Notice that the basic version of the RM that is described in Subsection 2.1 could have been used instead for the sake of example. The sampling frequency was $6 . \overline{6} \mathrm{~Hz}$. The tuning parameters of the RM are all collected in Table 3. See Table 1 for details on them. The additional tuning parameters concerning the path generation were $r_{c}^{\min }=10 \mathrm{~m}$ and $\rho_{c}=1$. They held for both pairs of twin clothoids. Table 4 gives the waypoints which sketch out the path. The tuning parameters of the MCS, regarding the controller, were

$$
\left\{\begin{array}{l}
\boldsymbol{K}_{\boldsymbol{P}}=\operatorname{diag}(52.6,83.8,53.3,69.2), \\
\boldsymbol{K}_{\boldsymbol{I}}=\operatorname{diag}(5,10,5,20), \\
\boldsymbol{K}_{\boldsymbol{D}}=\operatorname{diag}(420.3,533.8,432.5,182.0),
\end{array}\right.
$$

Table 3: Tuning parameters of the RM

\begin{tabular}{lcccccl}
\hline Parameter & $1 \mathrm{st}$ & $2 \mathrm{nd}$ & $3 \mathrm{rd}$ & 4 th & 5 th & Unit \\
\hline$L$ & $L_{1}$ & $L_{2}$ & $L_{3}$ & $L_{4}$ & $L_{5}$ & $\mathrm{~m}$ \\
$V_{d}$ & 0.4 & 0.25 & 0.4 & 0.25 & 0.4 & $\mathrm{~m} / \mathrm{s}$ \\
$V_{i}$ & 0 & 0.25 & 0.25 & 0.25 & 0.25 & $\mathrm{~m} / \mathrm{s}$ \\
$V_{f}$ & 0.25 & 0.25 & 0.25 & 0.25 & 0 & $\mathrm{~m} / \mathrm{s}$ \\
$T_{a}$ & 20 & 20 & 20 & 20 & 20 & $\mathrm{~s}$ \\
$T_{d}$ & 20 & 20 & 20 & 20 & 20 & $\mathrm{~s}$ \\
$\epsilon_{L}$ & 0.05 & 0.05 & 0.05 & 0.05 & 0.05 & - \\
$\theta_{a}$ & 0.8 & 0.8 & 0.8 & 0.8 & 0.8 & - \\
$\theta_{d}$ & 0.2 & 0.2 & 0.2 & 0.2 & 0.2 & - \\
$\theta_{0}$ & $\delta$ & $\delta$ & $\delta$ & $\delta$ & $\delta$ & - \\
\hline
\end{tabular}

where $L_{1}=L_{5} \approx 27.4457 \mathrm{~m}, L_{2}=L_{4} \approx 43.1760 \mathrm{~m}$, $L_{3} \approx 43.0579 \mathrm{~m}$, and $\delta=\exp (-13)$.

Table 4: Table of waypoints in the NE-plane

\begin{tabular}{lccccc}
\hline Waypoint & 1st & 2nd & 3rd & 4th & Unit \\
\hline North & 0 & 60 & 0 & 60 & $\mathrm{~m}$ \\
East & 0 & 0 & 90 & 90 & $\mathrm{~m}$ \\
\hline
\end{tabular}

and

$\left\{\begin{array}{l}\left(\boldsymbol{W}_{\boldsymbol{C}}\right)_{i, j}=\left(\boldsymbol{W}_{\boldsymbol{L}}\right)_{i, j}=\left(\boldsymbol{W}_{\boldsymbol{M}}\right)_{i, j}=\left(\boldsymbol{W}_{\boldsymbol{Q}}\right)_{i, j}=1, \quad \text { and } \\ \boldsymbol{W}_{\boldsymbol{P}}=\boldsymbol{I},\end{array}\right.$

where $i, j \in\{1,2,3,4\}$, and $\boldsymbol{I} \in \mathbb{R}^{5 \times 5}$ is an identity matrix, whereas the tuning parameters regarding the state observer, were

$$
\left\{\begin{array}{l}
\boldsymbol{L}_{\mathbf{1 1}}=\operatorname{diag}(0.1,0.1,10,10) \widehat{\boldsymbol{M}}^{-1}, \\
\boldsymbol{L}_{\mathbf{1 2}}=\operatorname{diag}(5,5,5,5) \widehat{\boldsymbol{M}}^{-1}, \\
\boldsymbol{L}_{\mathbf{2 1}}=\operatorname{diag}(0.03,0.03,3,3) \widehat{\boldsymbol{M}}^{-1}, \\
\boldsymbol{L}_{\mathbf{2 2}}=\operatorname{diag}(100,100,100,100) \widehat{\boldsymbol{M}}^{-1} \\
\epsilon=0.0005, \quad \text { and } \gamma=1,
\end{array}\right.
$$

where $\widehat{\boldsymbol{M}} \in \mathbb{R}^{4 \times 4}$ is the weighted nominal inertia matrix of the ROV implemented in the MCS. See Fernandes et al. (2015) for details on the tuning parameters.

Figures 10-13 are exclusively related to the path generated as reference. Figure 10 depicts the path based on the Table 4 . Figure 11 depicts the path curvature. Notice that $\kappa\left(\varpi_{p}(t)\right)$ is continuous, and that $\kappa\left(\varpi_{p}(t)\right)$ $\leqslant 1 / r_{c}^{\min }=0.1 \mathrm{~m}^{-1} \forall \varpi_{p}(t) \in[0,1] \wedge \forall t \in \mathbb{R}_{\geqslant 0}$. The parameter $\varpi_{p}(t)$, and its derivatives with respect to time, are shown in Figure 12. The whole path, whose length is $\approx 184.3 \mathrm{~m}$, has five parts, each of which with a characteristic function $\mathrm{h}_{i}\left(\varpi_{p}(t)\right)$, and parameterised by a parameter $\varpi_{i}(t), i \in\{1,2,3,4,5\}$, see Figure 13 . 


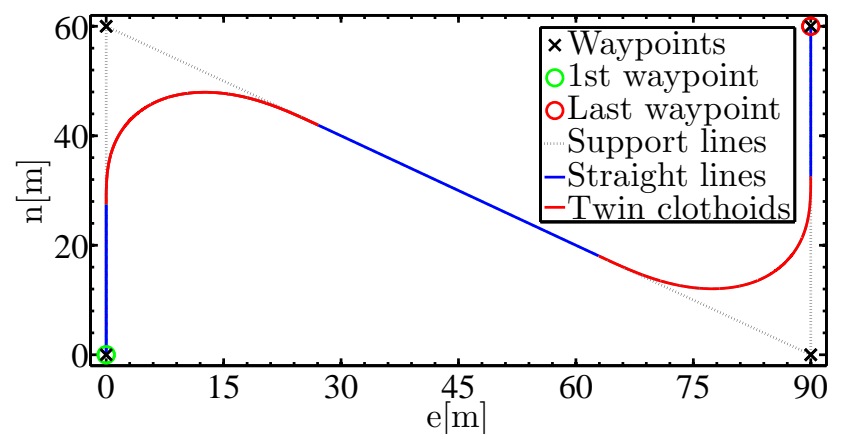

Figure 10: Reference path in the NE-plane.

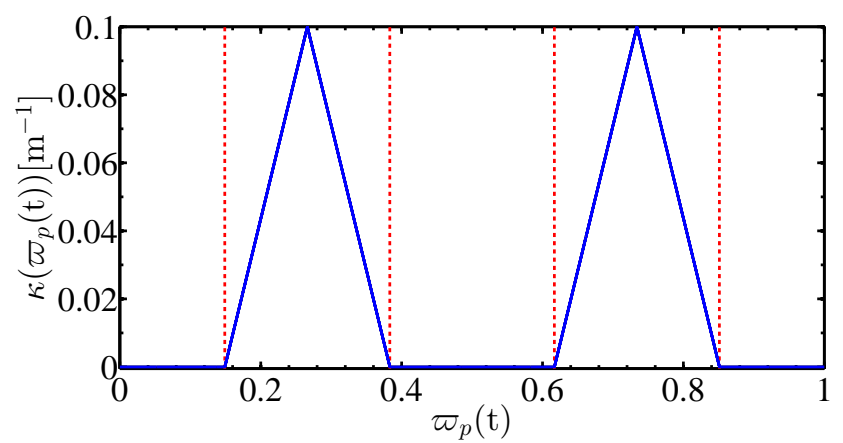

Figure 11: Reference path curvature.

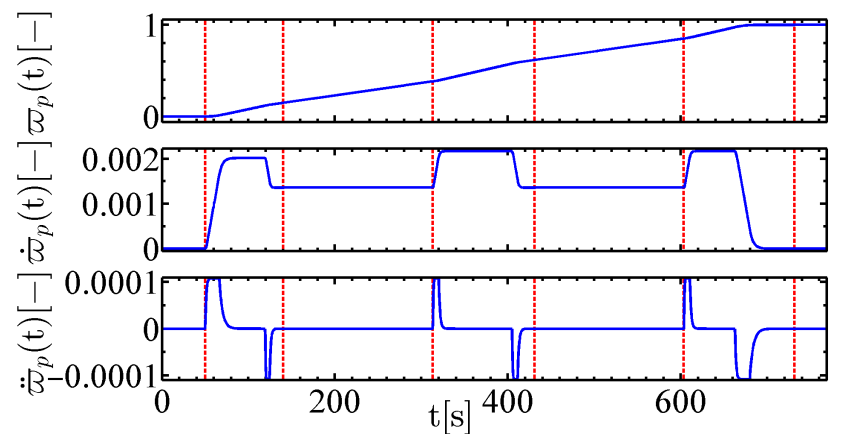

Figure 12: Parameter $\varpi_{p}(t)$ and its derivatives with respect to time $\dot{\varpi}_{p}(t)$ and $\ddot{\varpi}_{p}(t)$.

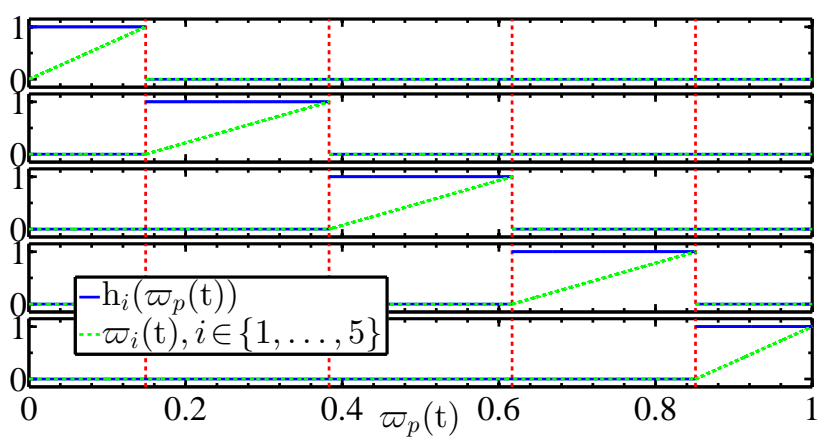

Figure 13: Characteristic functions and parametrisation of the five parts of the reference path.

The vertical dashed red lines separate each part of the path. The desired depth of $80 \mathrm{~m}$ was kept constant.

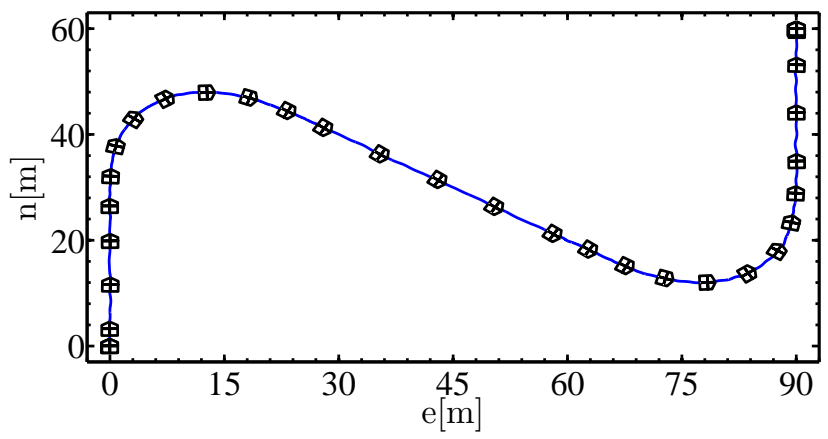

Figure 14: Trajectory described by the ROV in the NEplane. The patches indicate the heading.

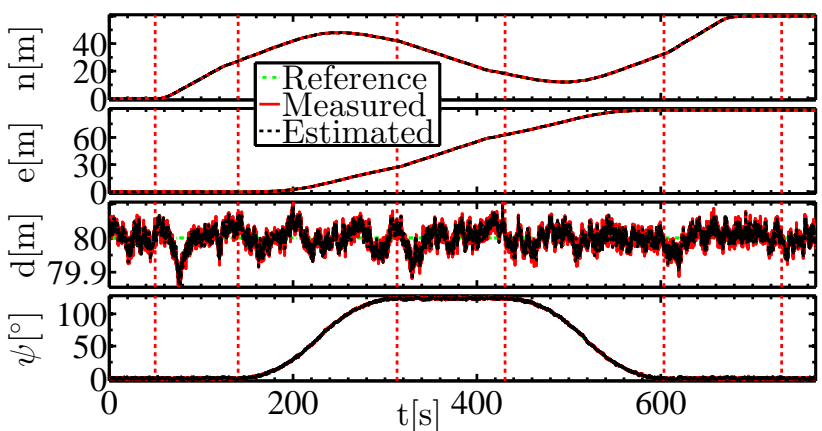

Figure 15: Position and heading (NED frame).

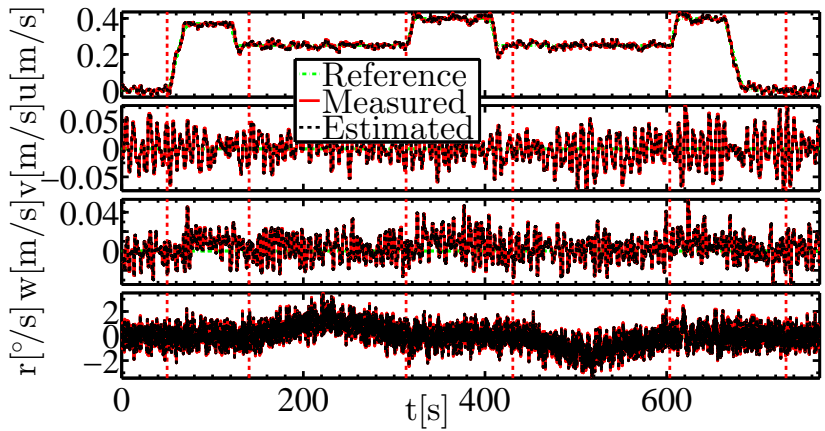

Figure 16: Linear and angular velocities (BF frame).

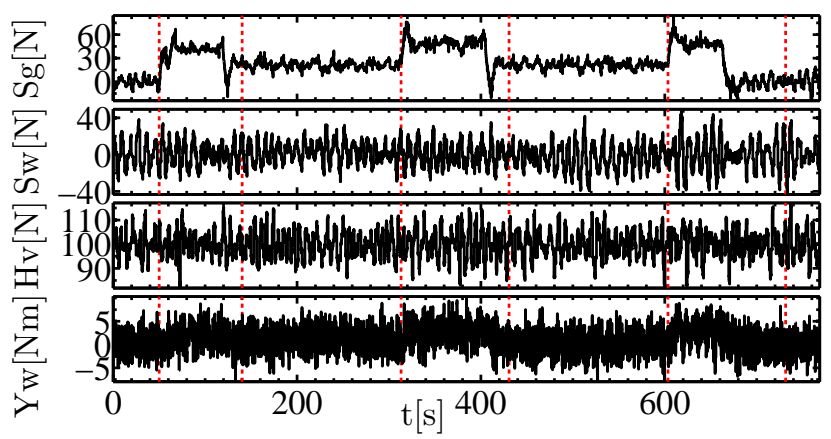

Figure 17: Commanded thrust forces and moment. Legend: $\mathrm{Sg}=$ Surge, $\mathrm{Sw}=\mathrm{Sway}, \mathrm{Hv}=$ Heave, and $\mathrm{Yw}_{\mathrm{w}}=$ Yaw.

Figure 14 depicts the trajectory described by Min- 
erva in the NE-plane. Notice the strong resemblance it bears to the reference path in Figure 10. The motion started at $50 \mathrm{~s}$ from DP, and stopped back in DP at $\approx 731.5 \mathrm{~s}$. Figure 15 depicts the described trajectory componentwise. The maximum absolute (spatial) position error is $<0.5 \mathrm{~m}$, where the maximum absolute depth error is $<0.15 \mathrm{~m}$. The maximum absolute heading error is $<2.5^{\circ}$. The satisfactory performance of the MCS under challenging operating conditions, e.g. motion concerning the three horizontal DoFs simultaneously, plant parameter variations, and measurement errors and noise, could be attained thanks to the suitable and sufficiently smooth motion references generated by the proposed path generation scheme. Figure 16 depicts the corresponding velocities along the trajectory. The measured and the estimated velocities remained close to the references all the time. It is worthwhile to realise that $v_{m}=V_{d}, v_{i}=V_{i}$, and $v_{f}=V_{f}$ in all four last parts of the path. In the first part, however, $v_{m}=0.93 V_{d}=0.372 \mathrm{~m} / \mathrm{s}$, according to the numerical method employed to determine $v_{m}$. The vertical dashed red lines in Figures 15-17 separate the parts, which began at the instants $50 \mathrm{~s}, \approx 140.3 \mathrm{~s}, \approx 313.1 \mathrm{~s}$, $\approx 430.6 \mathrm{~s}$, and $\approx 603.5 \mathrm{~s}$, respectively. The latter ends at $\approx 731.5 \mathrm{~s}$. The same division holds for Figure 12 . Figure 17 depicts the commanded thrust forces and moment from the propulsion system of the ROV. The curves somewhat mimic the shapes of the reference velocities depicted in Figure 16. When it comes to the sway and heave forces, and the yaw moment, the curves gently oscillate around constant values in order to compensate for disturbances. The heave thrust force kept the desired depth by counteracting the positive buoyancy force of $\approx 100 \mathrm{~N}$ of Minerva.

\section{Concluding remarks}

The paper dealt with the generation of sufficiently smooth references for guiding the motion of ROVs along purposefully pre-defined curvature-continuous paths. An RM that synthesises references concerning a single DoF motion was initially described. After that, the references synthesised by the RM were used to parameterise other references concerning the motion along curvature-continuous paths comprised of rectilinear and curvilinear parts. Both the proposed RM and the proposed path generation scheme proved to be able to synthesise references which yield ROV motion: i) under less induced plant parameter variations; ii) that favours energy saving along the motion; iii) with high overall accuracy; and iv) with finite convergence time. Moreover, both of them were easily tuned through the use of meaningful tuning parameters.

Future works must seek to find suitable numerical

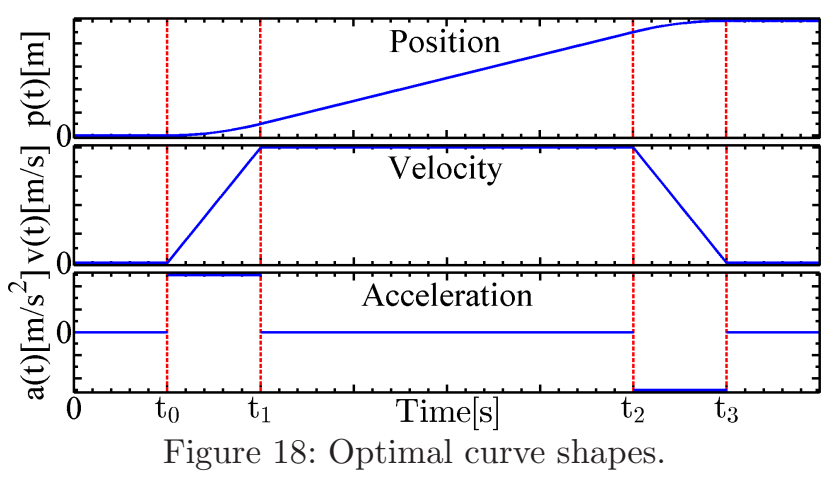

methods for determining the cruise velocity in the proposed advanced version of the RM. Other advanced versions can also be devised in the future, in order to serve yet different purposes. Also, spatial paths based on 3D spirals, e.g. as suggested by Harary and Tal (2012), must be treated.

\section{A. Optimal curve shapes}

Figure 18 shows the shapes of the optimal acceleration, velocity, and position curves along a straight line path which is to be traversed under the condition of constrained acceleration and velocity. The velocity curve is optimal in the sense that it yields the shortest travelling time $\mathrm{T}_{\min }:=\mathrm{t}_{3}-\mathrm{t}_{0}[\mathrm{~s}]$ between both path's extremities, which are separated by the distance $d \in \mathbb{R}_{>0}[\mathrm{~m}]$. The path is to be traversed under the additional constraints: i) $\mathrm{a}\left(\mathrm{t}_{0}\right)=\mathrm{v}\left(\mathrm{t}_{0}\right)=\mathrm{p}\left(\mathrm{t}_{0}\right)=0$; and ii) $\mathrm{a}\left(\mathrm{t}_{3}\right)=$ $\mathrm{v}\left(\mathrm{t}_{3}\right)=0$ and $\mathrm{p}\left(\mathrm{t}_{3}\right)=\mathrm{d}$. Symmetry between the maximum acceleration and deceleration is assumed for the sake of simplicity without loss of generality. Let these maximum values be equal to $\pm \mathrm{a}_{\max }$, where $\mathrm{a}_{\max } \in \mathbb{R}_{>0}$ $\left[\mathrm{m} / \mathrm{s}^{2}\right]$. Let, in addition, the maximum velocity be equal to $\mathrm{v}_{\max } \in \mathbb{R}_{>0}[\mathrm{~m} / \mathrm{s}]$. It is immediate to verify the claim that $\mathrm{v}(\mathrm{t})$ is optimal by realising that for any function $\mathrm{a}^{\prime}(\mathrm{t}): \mathcal{T} \rightarrow \mathbb{R}$, where $\mathrm{a}^{\prime}(\mathrm{t})$ is a continuous function defined on the compact set $\mathcal{T} \subset \mathbb{R} \mid \mathcal{T}:=\{\mathrm{t} \in$ $\left.\mathbb{R} \mid \mathrm{t} \in\left[\mathrm{t}_{0}, \mathrm{t}_{3}\right]\right\}$, such that $\left|\mathrm{a}^{\prime}(\mathrm{t})\right|<|\mathrm{a}(\mathrm{t})|$, the result

$$
\mathrm{p}^{\prime}\left(\mathrm{t}_{0}, \mathrm{t}_{3}\right)=\int_{\mathrm{t}_{0}}^{\mathrm{t}_{3}}\left(\int_{\mathrm{t}_{0}}^{\mathrm{t}_{3}} \mathrm{a}^{\prime}(\mathrm{t}) \mathrm{dt}\right) \mathrm{dt}=\mathrm{d}^{\prime}<\mathrm{d}
$$

is obtained. Hence, travelling times greater than $\mathrm{T}_{\min }$ are needed to eventually cover the entire distance $d$.

It is worth knowing that

$$
\begin{array}{r}
\mathrm{a}(\mathrm{t}):=\mathrm{a}_{\max }\left(\mathrm{H}\left(\mathrm{t}-\mathrm{t}_{0}\right)-\mathrm{H}\left(\mathrm{t}-\mathrm{t}_{1}\right)-\right. \\
\left.\mathrm{H}\left(\mathrm{t}-\mathrm{t}_{2}\right)+\mathrm{H}\left(\mathrm{t}-\mathrm{t}_{3}\right)\right),
\end{array}
$$


whereupon

$$
\begin{gathered}
\mathrm{p}\left(\mathrm{t}_{0}, \mathrm{t}_{1}, \mathrm{t}_{2}, \mathrm{t}_{3}\right)=\int_{\mathrm{t}_{0}}^{\mathrm{t}_{3}}\left(\int_{\mathrm{t}_{0}}^{\mathrm{t}_{3}} \mathrm{a}(\mathrm{t}) \mathrm{dt}\right) \mathrm{dt}= \\
\frac{\mathrm{a}_{\max }}{2}\left(\left(\mathrm{t}_{3}-\mathrm{t}_{0}\right)^{2}-\left(\mathrm{t}_{3}-\mathrm{t}_{1}\right)^{2}-\left(\mathrm{t}_{3}-\mathrm{t}_{2}\right)^{2}\right)=\mathrm{d} .
\end{gathered}
$$

The claim in this appendix can be alternatively formulated and proved in the framework of the calculus of variations (van Brunt, 2004; Forsyth, 1960).

\section{B. NTNU's ROV Minerva}

Minerva is a SUB-fighter 7500 ROV delivered by Sperre AS in 2003. The NTNU's Research Vessel (R/V) Gunnerus (http://www.ntnu.edu/marine/gunnerus) is the support vessel used to operate Minerva. The ROV is powered from, and communicates with, R/V Gunnerus through a $600 \mathrm{~m}$-long umbilical cable. Minerva has five thrusters with fixed pitch propellers. The starboard and port thrusters are oriented $10^{\circ}$ towards the longitudinal axis. The lateral thruster is the only that has one propeller at each end of its shaft, whereas all the other thrusters have a single propeller each. Figure 19 shows the thruster installation configuration. Table 5 keeps on the specifications of Minerva. Details can be found in Sørensen et al. (2012) and Dukan et al. (2011).

A high-precision hydroacoustic positioning system model HiPAP 500 by Kongsberg Maritime AS determines the north $n$ and east $e$ position components of Minerva relative to the $\mathrm{R} / \mathrm{V}$ Gunnerus with accuracy better than $0.1 \mathrm{~m}$ at update rates $\leqslant 1 \mathrm{~Hz}$. The depth $d$ (down position component), see Figure 2, is determined based on the measurements provided by the precision, temperature compensated, piezo-resistive underwater pressure sensor model miniIPS 0760001-100 by Valeport Ltd. It has full-scale span 100 bar, accuracy \pm 10 mbar, and resolution 1 mbar. Its maximum output update rate is $8 \mathrm{~Hz}$. The heading angle $\psi$ and the yaw rate $r$ are determined through the use of a dedicated complementary filter (Mahony et al., 2008) that treats the measurements provided by the Micro-Electro-Mechanical System (MEMS)-based Inertial Measurement Unit (IMU) model MTi-100 by Xsens Technologies B.V. The gyroscopes of the IMU have typical full-scale spans $450^{\circ} \mathrm{s}$, maximum bias repeatability $0.2^{\circ} / \mathrm{s}$ (one year), and typical in-run bias stability $10^{\circ} / \mathrm{h}$. Its accelerometers have typical full-scale spans $50 \mathrm{~m} / \mathrm{s}^{2}$, maximum bias repeatability $30 \mathrm{~mm} / \mathrm{s}^{2}$ (one year), and typical inrun bias stability $40 \mu \mathrm{g}$. The maximum output update rate is $2 \mathrm{kHz}$ with latency $<2 \mathrm{~ms}$. The velocities $u$, $v$, and $w$ are simultaneously measured by a $1200 \mathrm{kHz}$ Workhorse Navigator Doppler Velocity Log (DVL) by Teledyne RD Instruments, Inc. It has full-scale spans

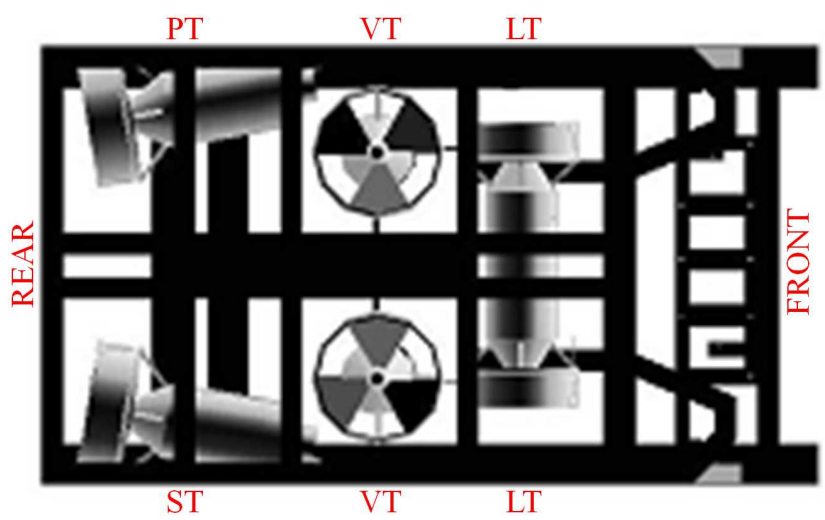

Figure 19: Thruster installation configurtion (top view). Legend: $\mathrm{L}=$ Lateral, $\mathrm{P}=$ Port, $\mathrm{S}=$ Starboard, $\mathrm{T}=$ Thruster, and $\mathrm{V}=$ Vertical.

Table 5: Basic specifications of Minerva

\begin{tabular}{ll}
\hline Dimensions & $1.44 \times 0.82 \times 0.81[\mathrm{~m}](\mathrm{L} \times \mathrm{W} \times \mathrm{H})$ \\
\hline Weight (air) & $485 \mathrm{~kg}$ \\
\hline Max. payload & $20 \mathrm{~kg}$ \\
\hline Max. depth & $700 \mathrm{~m}$ \\
\hline Thrusters & Horizontally installed: $3 \times 2 \mathrm{~kW}$ \\
& Vertically installed: $2 \times 2 \mathrm{~kW}$ \\
\hline & Surge: $-220 \mathrm{~N} / 480 \mathrm{~N}$ \\
Thrust capacity & Sway: $-195 \mathrm{~N} / 195 \mathrm{~N}$ \\
(min. / max.) & Heave: $-180 \mathrm{~N} / 390 \mathrm{~N}$ \\
& Yaw: $-300 \mathrm{Nm} / 300 \mathrm{Nm}$ \\
\hline
\end{tabular}

$10 \mathrm{~m} / \mathrm{s}$, long-term accuracy $\pm 0.2 \% \pm 1 \mathrm{~mm} / \mathrm{s}$, and resolution $1 \mathrm{~mm} / \mathrm{s}$. Its typical output update rate is $1 \mathrm{~Hz}$.

\section{Acknowledgments}

The present work is part of the work carried out at the Centre for Autonomous Marine Operations and Systems (AMOS) and at the Applied Underwater Robotics Laboratory (AUR-Lab). It is supported by The Research Council of Norway (NF) through the Centres of Excellence (SFF) funding scheme under the project No. 223254 - AMOS, and the project No. 192427.

\section{References}

Aguiar, A. and Pascoal, A. Modeling and control of an autonomous underwater shuttle for the transport of benthic laboratories. In IEEE OCEANS 1997 Canada. pages 888-895, 1997. doi:10.1109/OCEAN S.1997.624110. 
Aicardi, M., Cannata, G., Casalino, G., and Indiveri, G. Guidance of 3D underwater non-holonomic vehicle via projection on holonomic solutions. In $S U R T$ 2000 / WAC 2000 - USA. pages 11-16, 2000.

Åström, K. J. and Hägglund, T. PID controllers: theory, design, and tuning. Instrument Society of America, North Carolina, 2nd edition, 2011.

Åström, K. J. and Wittenmark, B. Computercontrolled systems: theory and design. Prentice Hall, Inc., Upper Saddle River, 2nd edition, 1997.

Breivik, M. and Fossen, T. I. Guidance laws for autonomous underwater vehicles. In A. V. Inzartsev, editor, Underwater vehicles, chapter 4, pages 51-76. I-Tech, Vienna, Austria, 2009.

van Brunt, B. The calculus of variations. SpringerVerlag, New York, 2004.

Caccia, M. Low-cost high-precision motion control for ROVs. In G. N. Roberts and R. Sutton, editors, $A d-$ vances in unmanned marine vehicles, volume 69 of IEE Control Series, chapter 9, pages 187-215. IEE - The Institution of Electrical Engineers, Stevenage, UK, 2006.

Caccia, M., Casalino, G., Cristi, R., and Veruggio, G. Acoustic motion estimation and control for an unmanned underwater vehicle in a structured environment. IFAC Control Engineering Practice, 1998. 6(5):661-670. doi:10.1016/S0967-0661(98)00057-4.

Caccia, M. and Veruggio, G. Guidance and control of a reconfigurable unmanned underwater vehicle. IFAC Control Engineering Practice, 2000. 8(1):2137. doi:10.1016/S0967-0661(99)00125-2.

Caharija, W. Integral line-of-sight guidance and control of underactuated marine vehicles. $\mathrm{PhD}$ thesis, Norwegian University of Science and Technology, Department of Engineering Cybernetics, 2014.

Caharija, W., Pettersen, K. Y., Sørensen, A. J., Candeloro, M., and Gravdahl, J. T. Relative velocity control and integral line of sight for path following of autonomous surface vessels: merging intuition with theory. Journal of Engineering for the Maritime Environment (Part M), 2014. 228(2):180-191. doi:10.11 $77 / 1475090213512293$.

Christ, R. D. and Wernli, R. L. The ROV manual: a user guide for observation class remotely operated vehicles. Butterworth-Heinemann, Oxford, UK, 2007.

Christ, R. D. and Wernli, R. L. The ROV manual: a user guide for remotely operated vehicles. Butterworth-Heinemann, Oxford, UK, 2nd edition, 2014.
Chyba, M., Haberkorn, T., Smith, R. N., and Choi, S. K. Design and implementation of time efficient trajectories for autonomous underwater vehicles. Ocean Engineering, 2008. 35(1):63-76. doi:10.1016/ j.oceaneng.2007.07.007.

Dukan, F. ROV motion control systems. PhD thesis, Norwegian University of Science and Technology, Department of Marine Technology, 2014.

Dukan, F., Ludvigsen, M., and Sørensen, A. J. Dynamic positioning system for a small size ROV with experimental results. In IEEE OCEANS 2011 Spain. pages 1-10, 2011. doi:10.1109/Oceans-Spain. 2011.6003399.

Egeland, O., Dalsmo, M., and Sørdalen, O. J. Feedback control of a nonholonomic underwater vehicle with constant desired configuration. The International Journal of Robotics Research, 1996. 15(1):24-35. doi: 10.1177/027836499601500102.

Farouki, R. T. Pythagorean-hodograph quintic transition curves of monotone curvature. Computer-Aided Design, 1997. 29(9):601-606. doi:10.1016/S0010-448 5(97)00004-3.

Farouki, R. T. Pythagorean-hodograph curves: algebra and geometry inseparable, volume 1 of Geometry and Computing. Springer-Verlag, Berlin, Germany, 2008.

Farouki, R. T. The Bernstein polynomial basis: a centennial retrospective. Computer-Aided Geometric Design, 2012. 29(6):379-419. doi:10.1016/j.cagd.201 2.03.001.

Farouki, R. T. and Sakkalis, T. Pythagorean hodographs. IBM Journal of Research and Development, 1990. 34(5):736-752. doi:10.1147/rd.345.0736.

Fernandes, D. A., Dukan, F., and Sørensen, A. J. Reference model for high performance and low energy consumption motions. In IFAC NGCUV 2012 Portugal. pages 217-222, 2012. doi:10.3182/2012041 0-3-PT-4028.00036.

Fernandes, D. A., Sørensen, A. J., Pettersen, K. Y., and Donha, D. C. Output feedback motion control system for observation class ROVs based on a highgain state observer: theoretical and experimental results. IFAC Control Engineering Practice, 2015. 39(0):90-102. doi:10.1016/j.conengprac.2014.12.005.

Forsyth, A. R. Calculus of variations. Dover Publications, Inc., New York, 1960. Originally published: Cambridge University Press, 1927. New York. 
Fossen, T. I. Handbook of marine craft hydrodynamics and motion control. John Wiley \& Sons Ltd., Chichester, UK, 2011.

Fossen, T. I. and Pettersen, K. Y. On uniform semiglobal exponential stability (USGES) of proportional line-of-sight guidance laws. IFAC Automatica, 2014. 50(11):2912-2917. doi:10.1016/j.automatica.2 014.10 .018 .

Guo, J., Chiu, F.-C., and Huang, C.-C. Design of a sliding mode fuzzy controller for the guidance and control of an autonomous underwater vehicle. Ocean Engineering, 2003. 30(16):2137-2155. doi:10.1016/S 0029-8018(03)00048-9.

Harary, G. and Tal, A. 3D Euler spirals for 3D curve completion. Computational Geometry, 2012. 45(3):115-126. doi:10.1016/j.comgeo.2011.10.001.

Healey, A. J. and Lienard, D. Multivariable slidingmode control for autonomous diving and steering of unmanned underwater vehicles. IEEE Journal of Oceanic Engineering, 1993. 18(3):327-339. doi:10.11 09/JOE.1993.236372.

Ho, G., Pavlovic, N., and Arrabito, R. Human factors issues with operating unmanned underwater vehicles. Proceedings of the Human Factors and Ergonomics Society Annual Meeting, 2011. 55(1):429433. doi:10.1177/1071181311551088.

Hsu, L., Costa, R. R., Lizarralde, F., and da Cunha, J. P. V. S. Dynamic positioning of remotely operated underwater vehicles. IEEE Robotics and Automation Magazine, 2000. 7(3):21-31. doi:10.1109/100.876 908.

Huo, F. and Poo, A.-N. Improving contouring accuracy by using generalized cross-coupled control. International Journal of Machine Tools \& Manufacture, 2012. 63(0):49-57. doi:10.1016/j.ijmachtools.2012.0 7.012 .

Kavraki, L. E. and LaValle, S. M. Motion planning. In B. Siciliano and O. Khatib, editors, Springer handbook of robotics, chapter 5, part A, pages 109-131. Springer-Verlag, Berlin, Germany, 2008.

Kumar, R. P., Dasgupta, A., and Kumar, C. S. Real-time optimal motion planning for autonomous underwater vehicles. Ocean Engineering, 2005. 32(11-12):1431-1447. doi:10.1016/j.oceaneng.2004. 11.010 .

Lai, X. C., Al-Mamun, A., and Ge, S. S. Polar polynomial curve for smooth, collision-free path generation between two arbitrary configurations for non- holonomic robots. In IEEE ISIC 2007 - Singapore. pages 59-64, 2007. doi:10.1109/ISIC.2007.4450861.

Landau, I. D. A survey of model reference adaptive techniques - theory and applications. IFAC Automatica, 1974. 10(4):353-379. doi:10.1016/0005-1098 (74)90064-8.

LaValle, S. M. Planning algorithms. Cambridge University Press, New York, 2006.

Lekkas, A. M. Guidance and path-planning systems for autonomous vehicles. $\mathrm{PhD}$ thesis, Norwegian University of Science and Technology, Department of Engineering Cybernetics, 2014.

Lekkas, A. M., Dahl, A. R., Breivik, M., and Fossen, T. I. Continuous-curvature path generation using Fermat's spiral. Modeling, Identification and Control, 2013. 34(4):183-198. doi:10.4173/mic.2013.4.3.

Levien, R. The Euler spiral: a mathematical history. Technical report UCB/EECS-2008-111, EECS Department, University of California, Berkeley, Berkeley, 2008. URL http://www.eecs.berkeley.edu/ Pubs/TechRpts/2008/EECS-2008-111.html.

Mahony, R., Hamel, T., and Pflimlin, J.-M. Nonlinear complementary filters on the special orthogonal group. IEEE Transactions on Automatic Control, 2008. 53(5):1203-1218. doi:10.1109/TAC.2008.9237 38.

Matsubara, A., Nagaoka, K., and Fujita, T. Modelreference feedforward controller design for highaccuracy contouring control of machine tool axes. CIRP Annals - Manufacturing Technology, 2011. 60(1):415-418. doi:10.1016/j.cirp.2011.03.029.

Minguez, J., Lamiraux, F., and Laumond, J.-P. Motion planning and obstacle avoidance. In B. Siciliano and O. Khatib, editors, Springer handbook of robotics, chapter 35, part E, pages 827-852. Springer-Verlag, Berlin, Germany, 2008.

Naeem, W., Sutton, R., Ahmad, S. M., and Burns, R. S. A review of guidance laws applicable to unmanned underwater vehicles. The Journal of Navigation, 2003. 56(1):15-29. doi:10.1017/S037346330200 2138.

Nelson, W. Continuous-curvature paths for autonomous vehicles. In IEEE ICRA 1989 - USA, volume 3. pages 1260-1264, 1989. doi:10.1109/ROBOT. 1989.100153.

Omerdic, E., Toal, D., Nolan, S., and Ahmad, H. ROV LATIS: next generation smart underwater vehicle. In 
G. N. Roberts and R. Sutton, editors, Further advances in unmanned marine vehicles, volume 77 of IET Control Engineering Series, chapter 2, pages 944. IET - The Institution of Engineering and Technology, Stevenage, UK, 2012.

Råde, L. and Westergren, B. Mathematics handbook for science and engineering. Studentlitteratur, Lund, Sweden, 5th edition, 2004.

Seidel, H.-P. An introduction to polar forms. IEEE Computer Graphics and Applications, 1993. 13(1):38-46. doi:10.1109/38.180116.

Slotine, J.-J. E. and Li, W. Applied nonlinear control. Pearson Education Taiwan Ltd., Taipei, Taiwan, 2005.

SNAME. The Society of Naval Architects and Marine Engineers. Nomenclature for treating the motion of a submerged body through a fluid. In Technical and research bulletin No. 1-5 - New York. pages 1-15, 1950.

Sørensen, A. J. Marine control systems: propulsion and motion control of ships and ocean structures. Lecture notes. Marine Technology Centre, Trondheim, Norway, 3rd edition, 2013.

Sørensen, A. J., Dukan, F., Ludvigsen, M., Fernandes, D. A., and Candeloro, M. Development of dynamic positioning and tracking system for the ROV Minerva. In G. N. Roberts and R. Sutton, editors, Further advances in unmanned marine vehicles, volume 77 of IET Control Engineering Series, chapter 6, pages 113-128. IET - The Institution of Engineering and Technology, Stevenage, UK, 2012.

Tsourdos, A., White, B., and Shanmugavel, M. Cooperative path planning of unmanned aerial vehicles. Aerospace Series. John Wiley \& Sons Ltd., Chichester, UK, 2011.

Vasilijević, A., Mišković, N., and Vukić, Z. Comparative assessment of human machine interfaces for ROV guidance with different levels of secondary visual workload. In IEEE MED 2013 - Greece. pages 1292-1297, 2013. doi:10.1109/MED.2013.6608886. 